\title{
A VLA 20 and 90 centimetre Radio Survey of Distant A-bell Clusters with Central cD Galaxies
}

\author{
Joydeep Bagchi \& Vijay K. Kapahi National Centre for Radio Astrophysics, TIFR, \\ Poona University Campus, Pune 411007
}

Received 1994 April 12; accepted 1994 May 27

\begin{abstract}
A radio survey, using the Very Large Array at 20 and $90 \mathrm{~cm} \lambda$ has been carried out in the direction of 46 distant Abell clusters $(0.1 \lesssim z \lesssim 0.3)$ dominated by a cD galaxy (clusters classified to be BautzMorgan I type). A radio source coincident with the $\mathrm{cD}$ galaxy was detected in 16 clusters. We find that the radio luminosity function of the $\mathrm{cD}$ galaxies at $20 \mathrm{~cm} \lambda$, and below the luminosity $P_{1.4 \mathrm{GHz}} \lesssim 10^{24.5} \mathrm{~W} \mathrm{~Hz}^{-1}$, is similar to that of brightest ellipticals in less clustered environments. Above this luminosity, the cDs seem to have a higher probability of becoming radio sources. The effect of optical brightness on radio emission is shown to be the same for the two classes. No significantly large population of verysteep-spectrum sources with spectral index $\alpha>1.2$ (flux density $\propto$ frequency ${ }^{-\alpha}$ ) was found to be associated with $\mathrm{cD}$ galaxies. A significant negative correlation is found between the radio luminosity of the $\mathrm{cD}$ galaxy and the cooling-time of the intra cluster medium near the galaxy. We also present evidence that the probability of radio emission from first-ranked galaxies is dependent upon their location relative to the geometrical centres of clusters and thus related to the morphological class and the evolutionary state of the clusters. We argue that both these effects are primarily caused by the dynamical evolution of these distant clusters of galaxies.
\end{abstract}

Key words: Clusters of galaxies - $\mathrm{cD}$ galaxies - radio luminosity functioncooling flows.

\section{Introduction}

Great clusters are the richest agglomerates of galaxies known where the space density of galaxies can surpass the average galaxian density by a large factor. Rich clusters can therefore serve as laboratories for the study of the complex interplay of galaxies with their environment. A striking example of the influence of the cluster environment on galaxies is the evidence of their morphological segregation, first mentioned by Hubble (1936) and Zwicky (1938) and later studied in greater detail by Oemler (1974), Melnick \& Sargent (1977) and Dressier (1980). These studies clearly demonstrated that the central regions of rich clusters are mostly populated by $\mathrm{E}$ and SO galaxies whereas their outlying and other low galaxy density regions are mainly spiral rich. Even more striking is the position of $\mathrm{cD}$ galaxies in 
clusters. Mathew, Morgan \& Schmidt (1964), while optically identifying the radio sources from early surveys, called attention to these extremely bright and massive galaxies of apparent elliptical morphology but with their associated amorphous and extensive stellar halos. The $\mathrm{cD}$ galaxies are almost always located near the densest and the innermost regions of rich clusters (Morgan \& Lesh 1965; Leir \& van Den Bergh 1977) although many relatively poor clusters are also known to contain these unique type of galaxies (Morgan et al. 1975; Albert et al. 1977). Despite these interesting observations, the physical mechanism causing their morphological segregation is not clearly known. The suggested mechanisms fall broadly under two categories. The first is the so called 'heredity' hypothesis according to which the morphologies of galaxies are decided at the time of their formation and remain unaltered subsequently. Thus ellipticals can and will form only in regions destined to become clusters. The second class of theories can be called the 'environment' theories which assume that all morphological types form in equal proportion everywhere and due to the influences of the local environment one galaxy type can evolve into another, eg. spiral galaxies may transform into SO galaxies if they lose their interstellar medium through ram-pressure ablations in intracluster gas (Gunn \& Gott 1972). A basic difficulty with the heredity model is the implication that the galaxies must have known what their present surroundings would be like at the time of their formation. This introduces obvious problems in the hierarchical clustering model of structure formation but could arise naturally in the adiabatic theory, where galaxy formation is regulated by the evolution of largescale structures.

From the radio astronomical point of view, the observed morphological segregation of galaxies in clusters has significant relevance. The powerful cosmic radio sources (radio luminosity at $1.4 \mathrm{GHz}, P_{1.4} \gtrsim 10^{22} \mathrm{WHz}^{-1}$ ) are mostly found associated with galaxies of $\mathrm{E}, \mathrm{SO}$ or $\mathrm{cD}$ types. The previous radio studies have indicated that amongst the clusters of all morphological types, the ones containing superbright elliptical galaxies near their cores (i.e. BM types I, II; Bautz \& Morgan 1970) are significantly more likely to contain radio sources associated with the brightest cluster members (eg. McHardy 1979). The exact physical mechanism causing this phenomenon is not clear but is possibly related to evolution of clusters. The supergiant $\mathrm{cD}$ galaxies appear to be the special products of dense environments as they are found at the densest regions of already dense cluster environments. The special structural, positional and kinematical properties of $\mathrm{cD}$ galaxies suggest that they may have originated and be evolving due to dynamical processes in clusters. The radio emission phenomenon from central galaxies might then also be affected by the evolution of their host clusters. Studies of their space distribution have shown that radio galaxies and quasars have undergone strong cosmological evolution. It is believed that powerful radio sources were more numerous in the past (Schmidt 1968; Ryle 1968) and were also pronouncedly smaller in their linear dimensions (eg. Kapahi 1989). It is believed that these effects are caused by a possibly denser medium surrounding these high redshift objects (eg. GopalKrishna \& Wiita 1991).

Yee \& Green (1987) have found that many radio-loud quasars at redshifts $z \approx 0.6$ are associated with clusters of galaxies comparable in richness to Abell clusters. However, such quasars do not occur in similar nearby clusters, thereby implying a drastic fading of quasar population since $z \approx 0.6$. It is expected that 'relics' of this past phase of activity are surviving in the form of radio sources associated with $\mathrm{E}$ and $\mathrm{cD}$ galaxies in clusters. An important goal of observational cosmology is to 
understand the physics governing the origin and evolution of optical and radio galaxies in the Universe. The evolution of galaxies in these wavebands may be intimately linked. Important clues to the understanding of physical mechanisms governing evolution can possibly be obtained by comparing the properties of clusters as a function of cosmological redshift. This requires a large-scale and complete catalogue of high redshift $(z \approx 1)$ Abell type clusters which unfortunately is not yet available, though some clusters upto $z=0.5$ are known (eg. Gunn et al. 1986). Very few systematic studies of the most distant A bell clusters $(z \approx 0.2)$ have so far been attempted though these form the largest sample of distant clusters known as yet.The main aim of the present paper is to systematically study the radio properties of a fairly large and complete sample of most distant Abell clusters (Distance class D = 5,6 or $0.1 \lesssim z \lesssim 0.3$ ) known to contain $\mathrm{cD}$ galaxies in their central regions. No systematic radio survey of Abell clusters of such distances and morphology is available at present. Recently Ball et al (1993) have reported the results of a 6 centimeter VLA survey of about 90 Abell clusters of cDClass of a lower redshift range $(z<0.10)$.

In the present paper the results of our analysis of radio properties of central cD galaxies in distant Abell clusters are reported. The full catalogue and details of the nature of other radio sources in the direction of these clusters would be presented in a subsequent paper (under preparation). The section-wise division of the present paper is as follows. Section 2 contains the details of sample of clusters selected for study. Observations and the data reduction procedure are described in section 3. Section 4 presents the radio images, and the basic radio and optical data. The scientific results and their discussion are presented in sections 5,6,7, and 8, where we also discuss the possibility of further future work and in section 9, the main conclusions of this study are summarised. We have assumed an Einstein-de Sitter Cosmology $\left(\Omega=1, q_{0}=0.5\right)$ and $50 \mathrm{~km} \mathrm{sec}^{-1} \mathrm{Mpc}^{-1}$ for the Hubble constant. Thus, 1 second of arc corresponds to $3.3 \mathrm{Kpc}$ at the redshift $z=0.15$.

\section{Sample selection}

We attempted to study a fairly large statistical sample of Abell clusters containing $\mathrm{cD}$ galaxies. All of the clusters observed were selected from amongst those in the largest distance classes $(D \geqslant 5)$ of Abell's catalogue. The main reasons for this selection criterion were as follows. First, due to an intrinsic rarity of clusters dominated by a giant $\mathrm{cD}$ galaxy (Bautz-Morgan Class I), a large statistical sample can only be formed by going to the most distant clusters in Abell's catalogue. Secondly, the. angular size subtended by distant clusters is comparatively smaller, enabling us to map the whole cluster field without a large loss of detection sensitivity caused by the primary beam attenuation of radio dishes. The relatively smaller angular extents of distant clusters also imply a smaller probability of a chance coincidence of an unrelated source in the direction of the cluster at a constant flux density level. Lastly, the clusters selected should not be biased in any way towards their radio or X-ray properties. The only safe way of ensuring this is to select clusters from optical catalogues, as was done here. The clusters were selected from the listing of Leir \& van Den Bergh (1977) who have classified all the clusters included in the original Abell's statistical sample into the Bautz-Morgan classes (see Bautz \& Morgan 1970 for details). The clusters so selected are: 
- Classified as BM type I or I: (i.e. contain a giant, first ranked $\mathrm{cD}$ galaxy).

- North of declination $\delta=-27^{\circ}$ (the limit of Palomar Plates).

- Classified to have a distance class 5 or larger, corresponding to estimated redshift range $0.1 \lesssim z \lesssim 0.3$ (as derived by Leir \& van Den Bergh 1977).

- Of a richness class $\mathrm{R} \geqslant 0$.

The 49 clusters satisfying these criteria were selected for observations with the VLA at $20 \mathrm{~cm}$ and $90 \mathrm{~cm}$ wavelengths.

\section{Observations and data reductions}

The observations were carried out with the VLA radio telescope (Napier, Thompson \& Ekers 1983). The array was in its C/D configuration during $20 \mathrm{~cm}$ observations (04 April 1988) and in the A/B configuration (17 February 1989) for the $90 \mathrm{~cm}$ observations. Due to constraints of available time and scheduling, 46 of the 49 clusters selected were actually observed at $20 \lambda \mathrm{cm} 2$. Only 34 of these could be observed also at $90 \mathrm{~cm} \lambda$ The observations were made with a bandwidth of $50 \mathrm{MHz}$ at $20 \mathrm{~cm}$ (centre frequencies at $1452.4 \mathrm{MHz}$ and $1502.4 \mathrm{MHz}$ ) and $3.13 \mathrm{MHz}$ at $90 \mathrm{~cm}$ (centered at 333.0 MHz and 327.5 MHz). The centre of each cluster was tracked by the telescopes in the array, on the average for a total duration of about 15 minutes. During this interval visibilities on all baselines were averaged for 30 seconds to obtain $\approx 10^{4}$ visibilities for each cluster. Nearby strong point sources were observed roughly every half an hour to serve as phase calibrators.

The observations were bootstrapped to the flux density scale of Baars et al. (1977) using either $3 \mathrm{C} 286$ or $3 \mathrm{C} 48$ as the primary flux density calibrators. Subsequent to initial calibration, the $\mathrm{u}-\mathrm{v}$ data were edited to remove any discrepant amplitude points. Data in the two IFs were then combined and the visibilities Fourier transformed and the point spread function deconvolved from the maps using the AIPS task MX. Two to three passes of phase self-calibration were performed to correct the visibility phases before the final images were produced. The final maps were of size $512 \times 512$ pixels $(\approx 50 \operatorname{arcmin} \times 50$ arcmin $)$ at $20 \mathrm{~cm}$ and $1024 \times 1024$ pixels $\left(\approx 3.4^{\circ} \times 3.4^{\circ}\right)$ at $90 \mathrm{~cm}$. These large map sizes were necessary to remove the effects of sidelobes of all the sources within the primary beam area. The final maps at $20 \mathrm{~cm} \lambda$ were made by convolving the clean components with circular Gaussian beams of 30" $\times 30^{\prime \prime}$ FWHM size (with 30\% visibility tapering at longest baselines). The full resolution of $90 \mathrm{~cm}$ observations could not be achieved due to a lack of necessary computing power. Therefore, the final resolution of the $90 \mathrm{~cm}$ images was $60^{\prime \prime} \times 60^{\prime \prime} \mathrm{FWHM}$, achieved after heavily tapering the measured visibilities. The list of clusters observed at the two frequencies is given in the first column of Table 1.

The observed r.m.s noise fluctuation on the final $20 \mathrm{~cm}$ maps was generally between 0.05 and $0.15 \mathrm{mJy} / \mathrm{beam}$ which is close to the expected level of thermal noise. The $90 \mathrm{~cm}$ maps generally had r.m.s noise levels of about 1 to $2 \mathrm{mJy} / \mathrm{beam}$, considerably higher than the expected thermal noise. This is mainly caused by the presence of many strong sources within the wide field of view and due to closure phase errors at $90 \mathrm{cms}$. We have adopted a conservative $12 \sigma$ signal requirement for $100 \%$ completion for unresolved radio sources near the image centres. This then implies that most $20 \mathrm{~cm}$ images are complete down to $\sim 1 \mathrm{~m}$. Ty whereas, most $90 \mathrm{~cm}$ images are 
complete down to $\sim 15-25 \mathrm{mJy}$. The detailed radio maps and the data on all the sources detected in each field will be presented in a separate paper. Here we concentrate on those radio sources that were found to be associated with $\mathrm{cD}$ galaxies. In order to find such sources the optical positions of the $\mathrm{cD}$ galaxies present in the clusters studied were measured on the POSS (Palomar Observatory Sky Survey) E-plates (red filter) using the digital $\mathrm{X}-\mathrm{Y}$ measuring machine available with the IR/X-ray astronomy group at TIFR, Bombay. We used SAO standard stars near the fields of interest for astrometry. The accuracy of optical positional measurement has an r.m.s $\left(\sigma_{0}\right)$ error range of $\approx(0.5-4.0)$ arcsec. We consider a radio source to be identified with a cD galaxy if the radio-optical positional offset $\left(\Delta_{R}-0\right)$ is less than 2.5 times the combined errors from radio and optical positional measurements, i.e. $\Delta_{R-0} \leqslant 2.5 \times \sigma_{R-0}$

$$
\text { where } \sigma_{R-O}=\left[\left(\sigma_{0}\right)^{2}+\left(\sigma_{R}\right)^{2}\right]^{1 / 2}
$$

Here $\sigma_{R}$ denotes the r.m.s error in radio position from the higher resolution $20 \mathrm{~cm}$ maps (generally about $1 "$ arc). Optical positions of the $\mathrm{cD}$ galaxies in 6 Abell clusters in this sample could not be measured as the corresponding sky survey prints were not available at Bombay. The optical data on one of these, viz. A1597 was however available from Huchra et al. (1990).

\section{Radio and optical data on $\mathrm{cD}$ galaxies}

Amongst the 41 clusters for which we had the optical data, $16 \mathrm{cD}$ galaxies were detected at the wavelength of $20 \mathrm{~cm} \lambda$ Radio maps of the 11 resolved sources (LAS $\approx 10$ arcsec) at $1.4 \mathrm{GHz}$ are presented in Fig. 1 which also contains maps of $4 \mathrm{cDs}$ that were resolved at $330 \mathrm{MHz}$ as well. Table 1 presents the radio and optical data on the 46 clusters that formed our sample. A blank in certain columns denotes non availability of data. The table is arranged as follows:

Col. 1: The Abell cluster number. An asterisk denotes the non availability of the optical position of the $\mathrm{cD}$ galaxy.

Col. 2 and 3: $\quad$ The optical R.A. and Dec. of the cD galaxy in the cluster (epoch 1950.0).

Col. 4 and 5: The flux density of the $\mathrm{cD}$ at 20 and $90 \mathrm{~cm}$ wavelengths respectively.

Col. 6: The distance of the $\mathrm{cD}$ galaxy from the cluster centre as listed in Abell's catalogue, measured in units of Abell radius $\left(\mathrm{R}_{A}\right)$.

Col. 7: Logarithm of the $1400 \mathrm{MHz}$ radio luminosity of the $\mathrm{cD}$ galaxy in units of $\mathrm{WHz}^{-1}$. If no spectral index information is available, a spectral index $\alpha=0.75$ has been assumed.

Col. 8, 9,10 and 11: Morphological data on the parent clusters. The redshifts for 20 clusters are based on spectroscopic measurements,(Huchra, privatecommunication), for the other clusters we have used the redshifts estimated by Leir \& Van Den Bergh (1977). These estimated values are enclosed in brackets. The BM classification, the distance class (D), and the richness class (R) were taken from the catalogue of Leir \& Van Den Bergh (1977). 


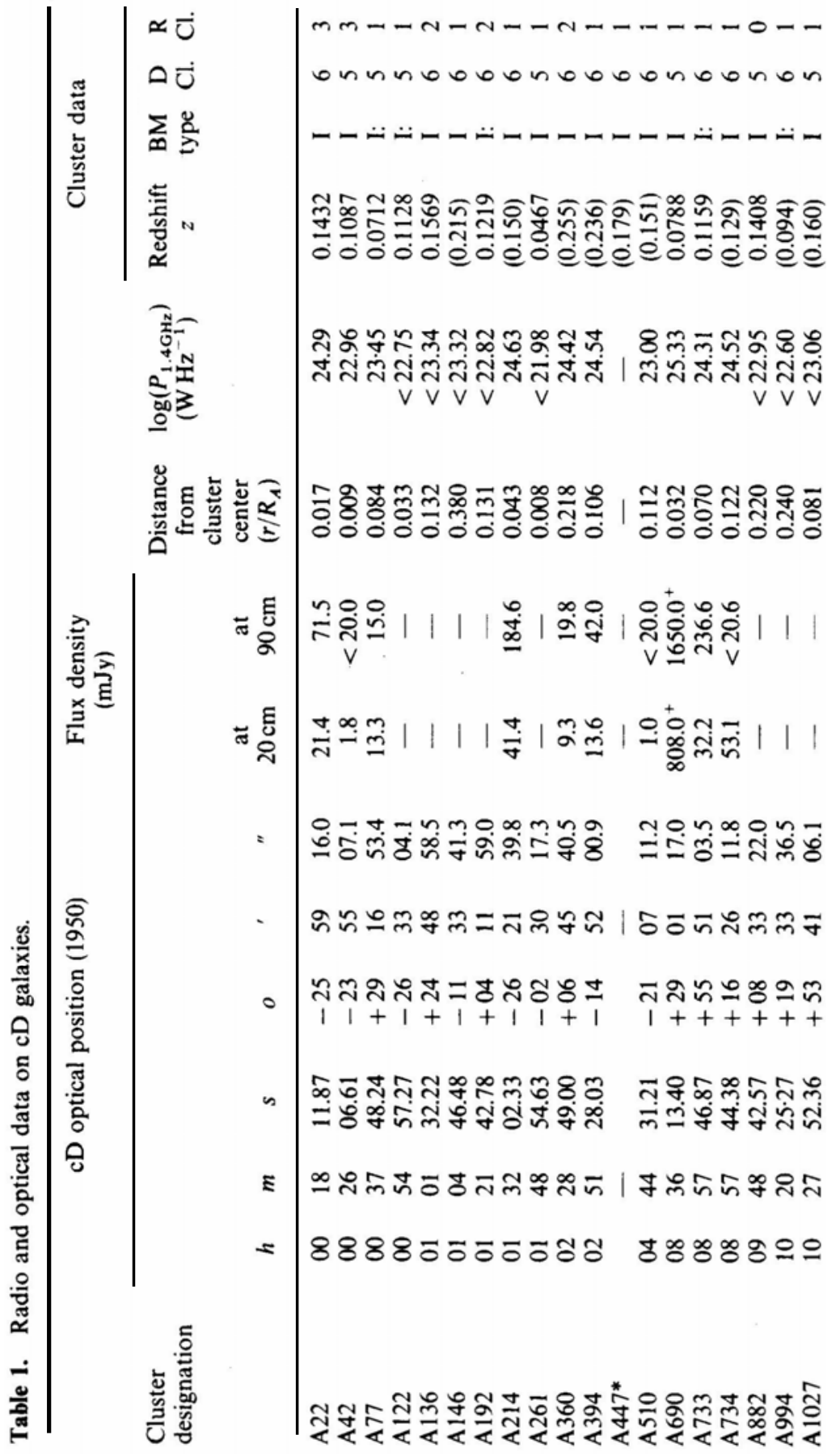




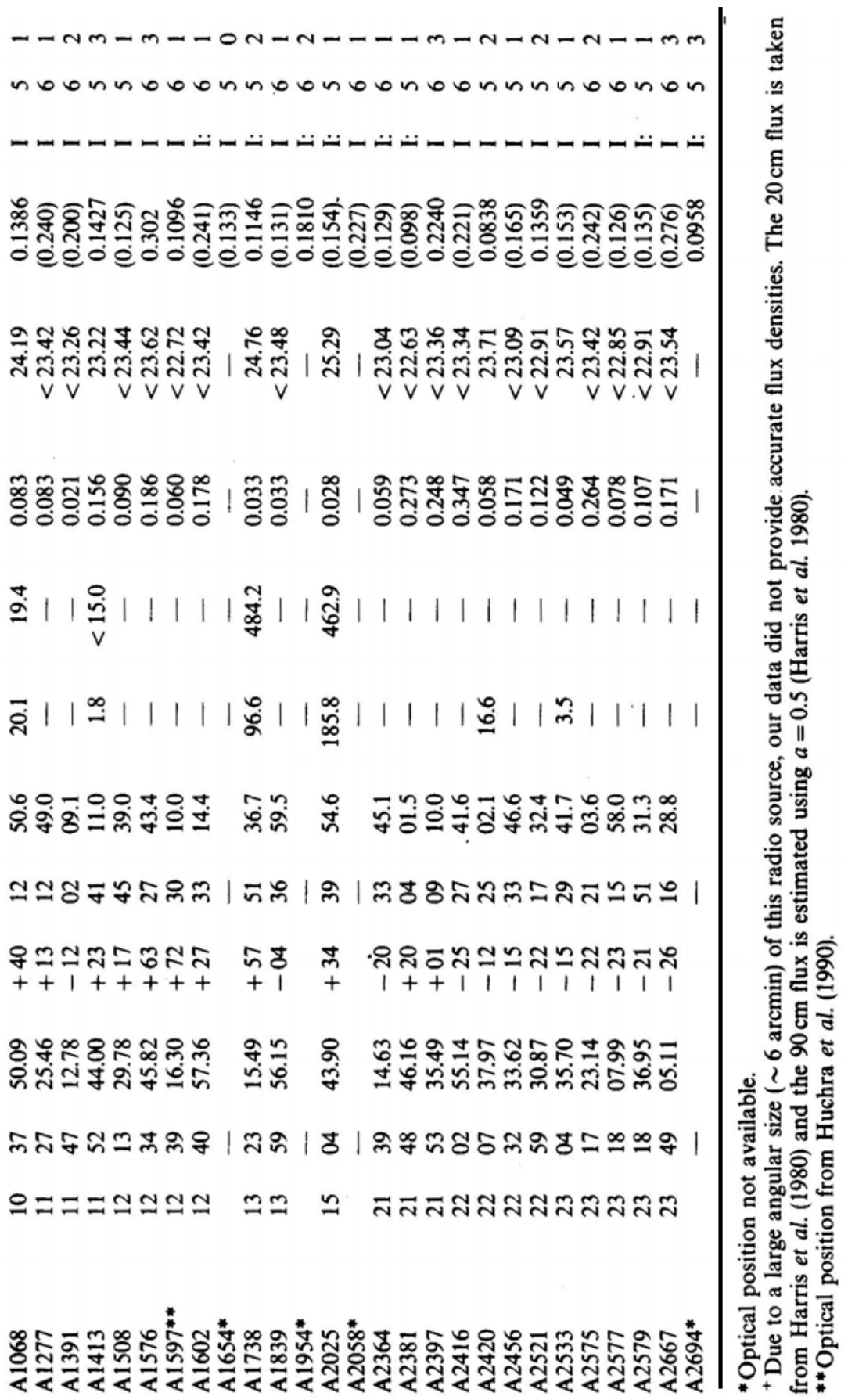



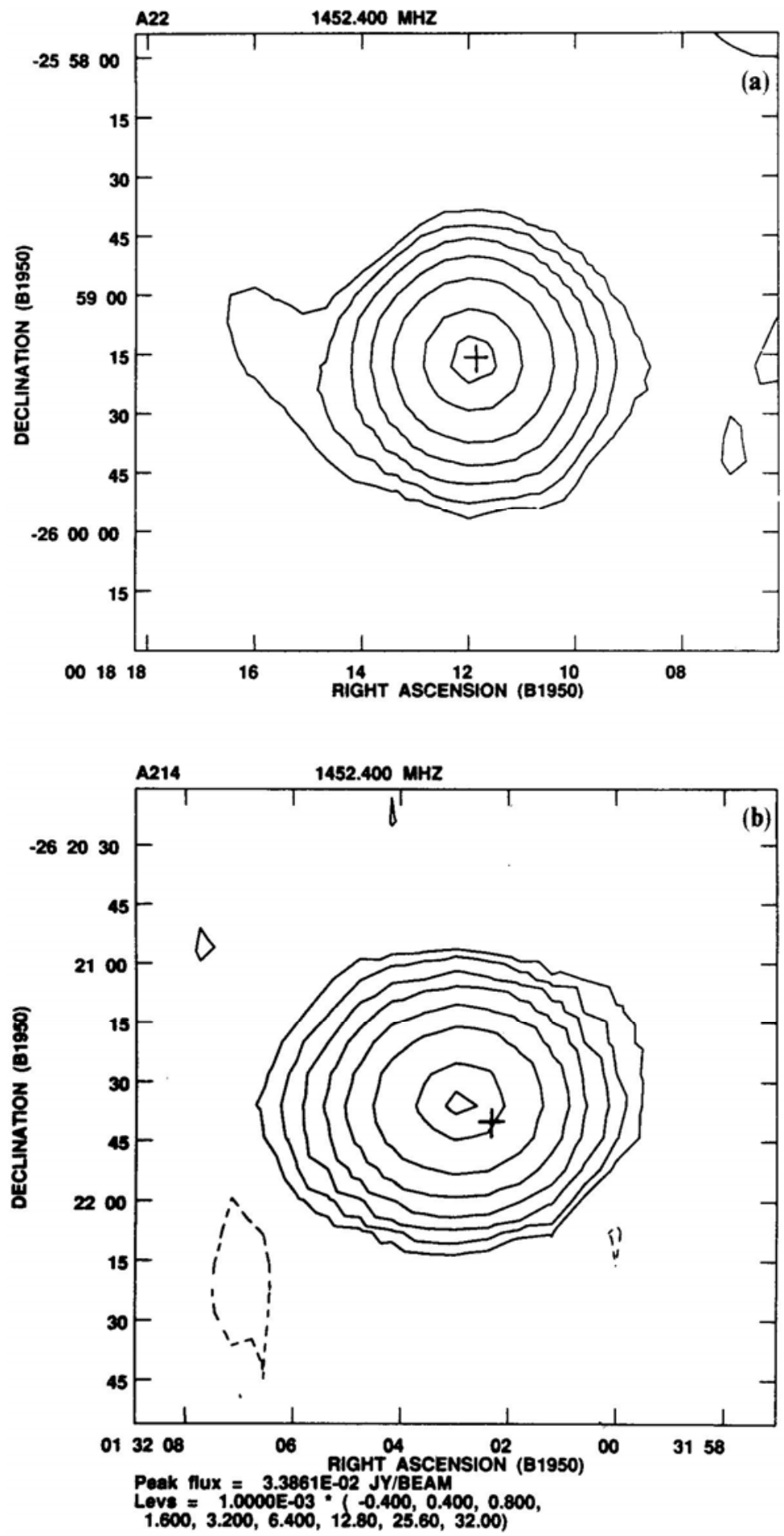

Figure 1.(a,b). 


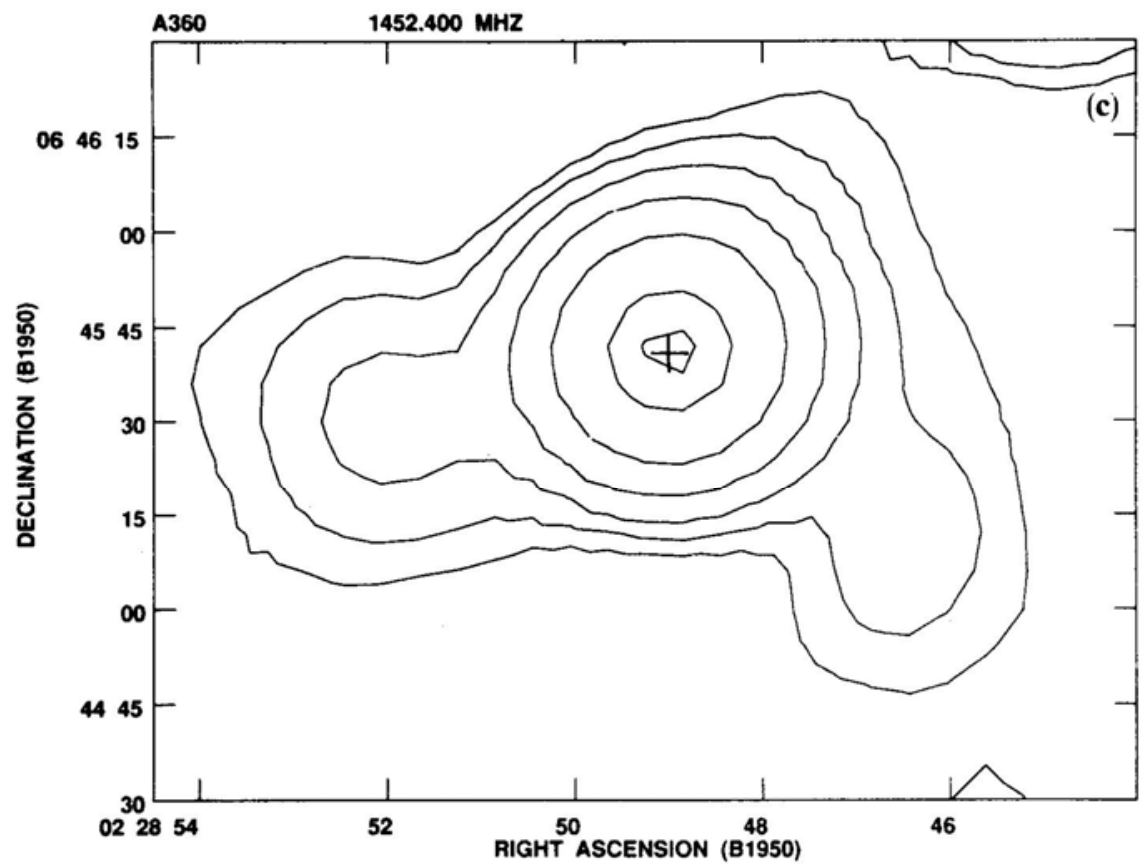

Levs $=1.0000 \mathrm{E}-03 \cdot(-0.200,0.200,0.400$,

$0.800,1.600,3.200,6.400,8.000$ )

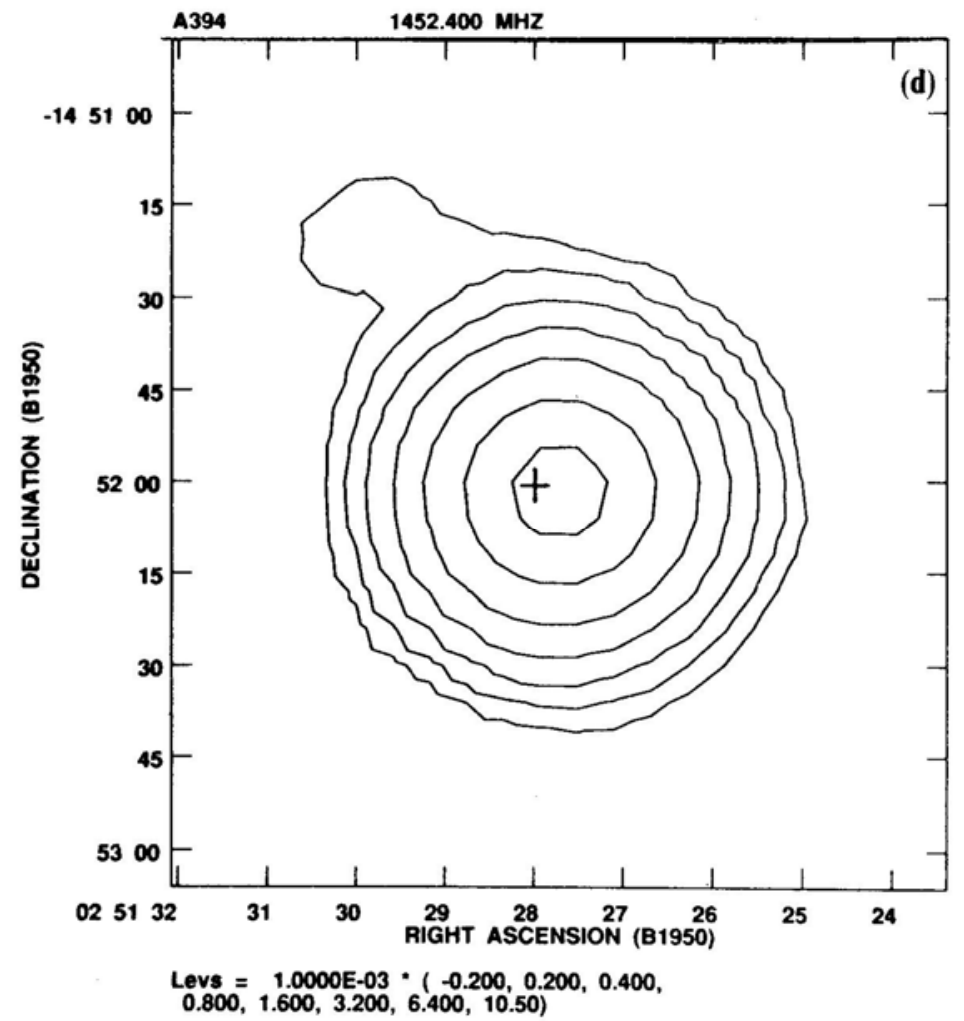

Figure $1(c, d)$. 


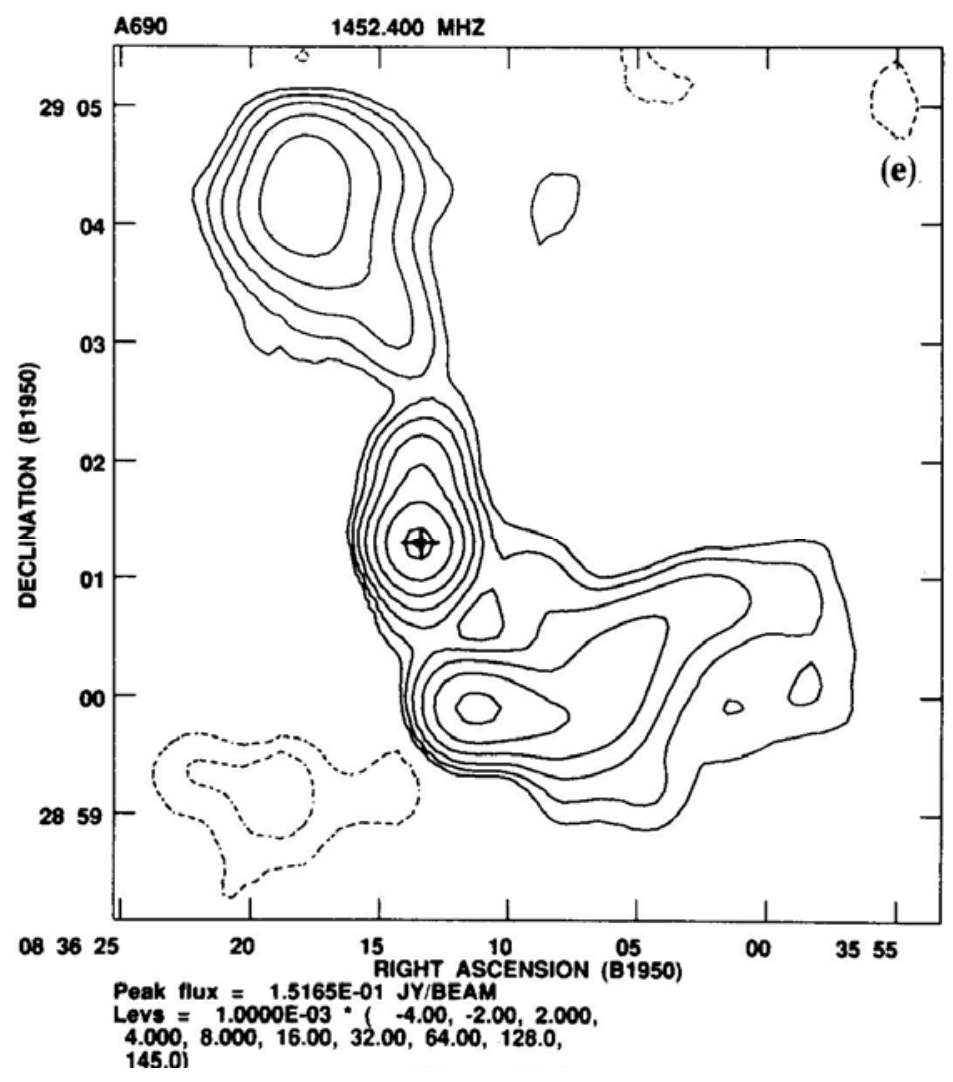

Figure 1 (e).

\section{The radio luminosity function of $\mathrm{cD}$ galaxies}

The radio luminosity function (RLF) of a class of objects is useful in estimating the probability of energy emission across a wide range of radio luminosities and can be used to study the effects of variable environmental factors on this probability. Here we construct the $1.4 \mathrm{GHz}$ fractional RLF of cD galaxies, using the information given in Table 1 . We use only the 41 clusters in which the optical positions of the $\mathrm{cD}$ galaxies were available. The estimated probability that a $\mathrm{cD}$ galaxy would be found to have a luminosity within the range $P_{1.4}$ to $P_{1.4}+\mathrm{d} P_{1.4}$ (where $P_{1.4}$ is the radio luminosity in units of $\mathrm{WHz}^{-1}$ at $1.4 \mathrm{GHz}$ ) is the so called differential radio luminosity function (DRLF), denoted by $\mathrm{dF}\left(P_{1.4}\right)$. If the function $\mathrm{dF}\left(P_{1.4}\right)$ is integrated over all luminosities greater than a certain value $P_{1.4}$ [here denoted by $\mathrm{F}\left(>P_{1.4}\right)$ ], obtain the integral radio luminosity function (IRLF). Our flux density detection limit of $\sim 1 \mathrm{mJy}$ in most of the clusters and the lower redshift limit of $\sim 0.05$ in our sample, essentially decide the weakest radio luminosity that can be reliably studied. This luminosity is in the range of $(21.8-22.2) \mathrm{WHz}^{-1}$ in $\log P_{1.4}$. We construct the fractional DRLF by initially subdividing the radio luminosities into differential intervals of width $\Delta\left(\log P_{1.4}\right)=0.4$. We then calculate, for each differential interval, the ratio of the number of cDs detected to the number that would have been detectable in our survey. This ratio is the DRLF for the given interval. 


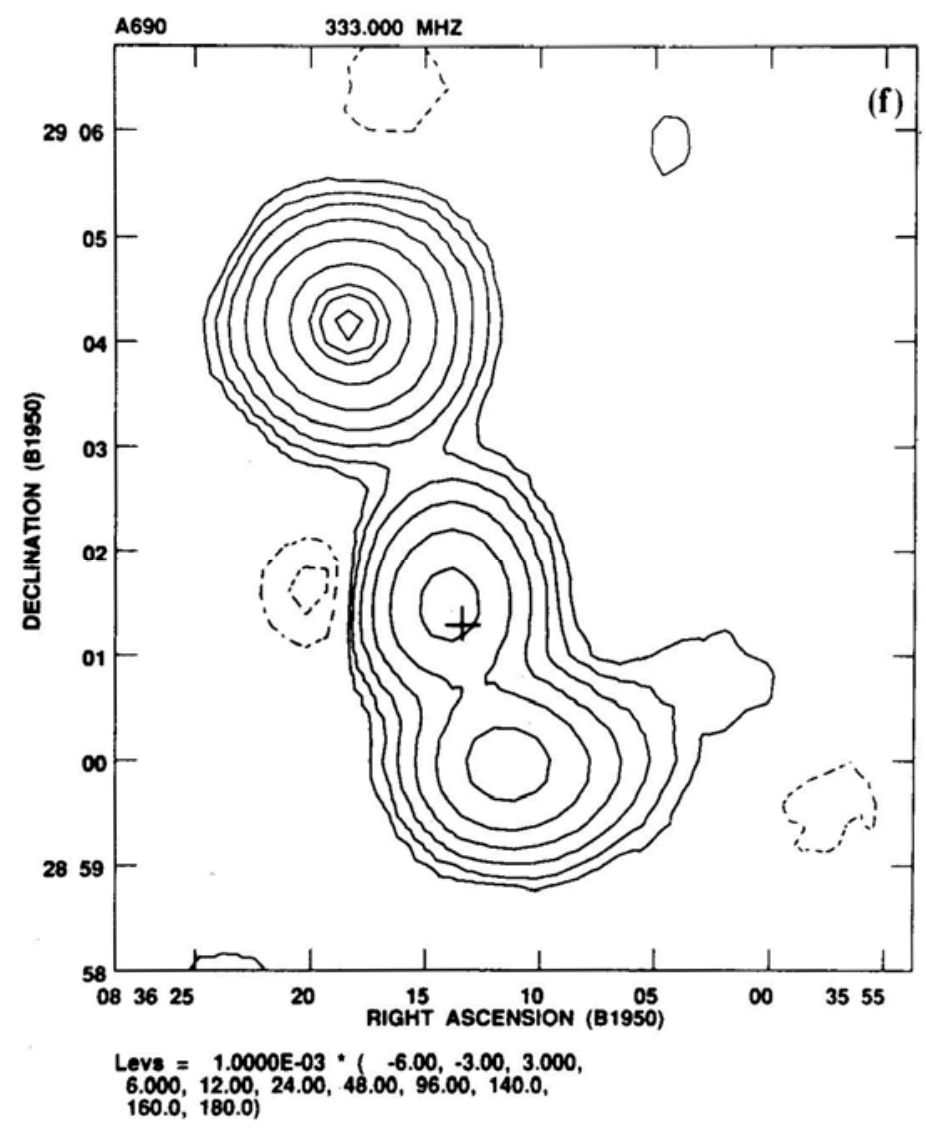

Figure 1 (f).

Table 2 summarises the information on the estimated DRLF and IRLF of cD galaxies observed by us. The graphical forms of these functions are shown in Fig. 2(a) and Fig. 2(b). The errors of estimating DRLF are assumed to be the Poissonian counting errors and the error bars on the IRLF values are obtained by quadratic combination of errors of DRLF. A fairly sharp change in the slope of the IRLF near $\log P_{1.4} \approx 24.5$ is visible in Fig. 2(b). This change in the slope of RLF at $\approx 10^{24.5} \mathrm{WHz}^{-1}$ is also a general behaviour of radio sources associated with bright elliptical galaxies outside rich clusters (Colla et al. 1975; Auriemma et al. 1977). Auriemma et al. (1977) found that irrespective of the optical magnitude of the galaxy, a 'break' occurs at a luminosity between $10^{24-25} \mathrm{WHz}^{-1}$. A comparison of $\mathrm{cD}$ galaxy luminosity function with the local radio luminosity function of brightest ellipticals in absolute photographic magnitude ranges $-22<\mathrm{Mp}<-21$ and $-21<\mathrm{Mp}<-20$ (from Auriemma et al. 1977), shows that below.the 'break'at $\approx 10^{24.5} \mathrm{WHz}^{-1}$ the cD galaxy function is quantitatively similar to the RLF of brighter ellipticals within errors (see Fig. 2b). However, above the 'break', the brighter elliptical galaxy function appears to be consistently below the RLF of cDs. We also find that the RLF of cDs, when compared with RLF of ellipticals in the magnitude range $-21<$ $\mathrm{Mp}<-20$, clearly indicate a higher probability of radio.emission for all $P_{1.4}>10^{22}$ 


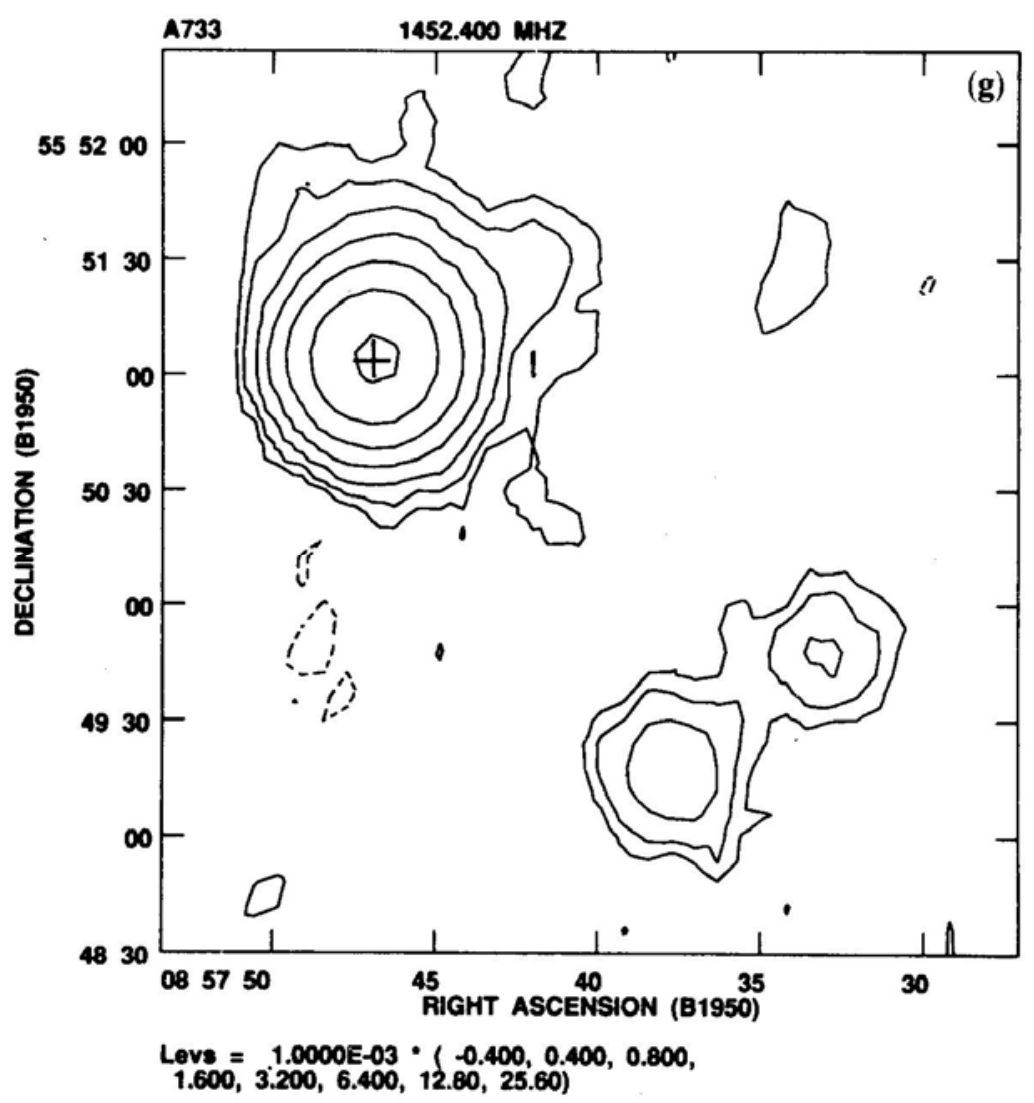

Figure 1 (g).

$\mathrm{WHz}^{-1}$ (Fig. 2b). For $\log P_{1.4}>24.8$ the estimated probability of radio emission is found to be $\approx 10 \pm 5 \%$ for $\mathrm{cD}$ galaxies, whereas it is only $\approx 3 \pm 1 \%$ and $\approx 0.8 \pm 1 \%$ in the case of E galaxies in the two magnitude intervals shown in Fig. 2b. Thus it is possible that beyond the 'break' at $\approx 10^{24 \cdot 5} \mathrm{WHz}^{-1}$, cDs in rich clusters have a somewhat higher probability of being radio sources than that of brightest ellipticals in less-dense environments. Unfortunately, the number of detectable radio sources emitting above $\log P_{1.4}>25$, is quite small and hence this result needs to be checked using a larger sample of sources.

For giant $\mathrm{E}$ galaxies located outside rich cluster environments, a correlation between the probability of radio emission and the optical brightness of the parent galaxy is well known (Colla et al 1975; Auriemma et al 1977). We have investigated the possibility that the higher probability of radio emission in $\mathrm{cDs}$ is related to their larger optical luminosity. The mean absolute visual magnitude of $\mathrm{cD}$ galaxies, $<M_{v}>_{\mathrm{CD}}$ was found to be -23.71 by Sandage (1976). This is converted into mean photographic magnitude $\left\langle M_{p}\right\rangle_{\mathrm{cD}}$ using the relation used by Auriemma et al (1977) to obtain,

$$
<M_{p}>_{\mathrm{cD}}=<M_{v}>_{\mathrm{cD}}+1.15=-22.56 .
$$




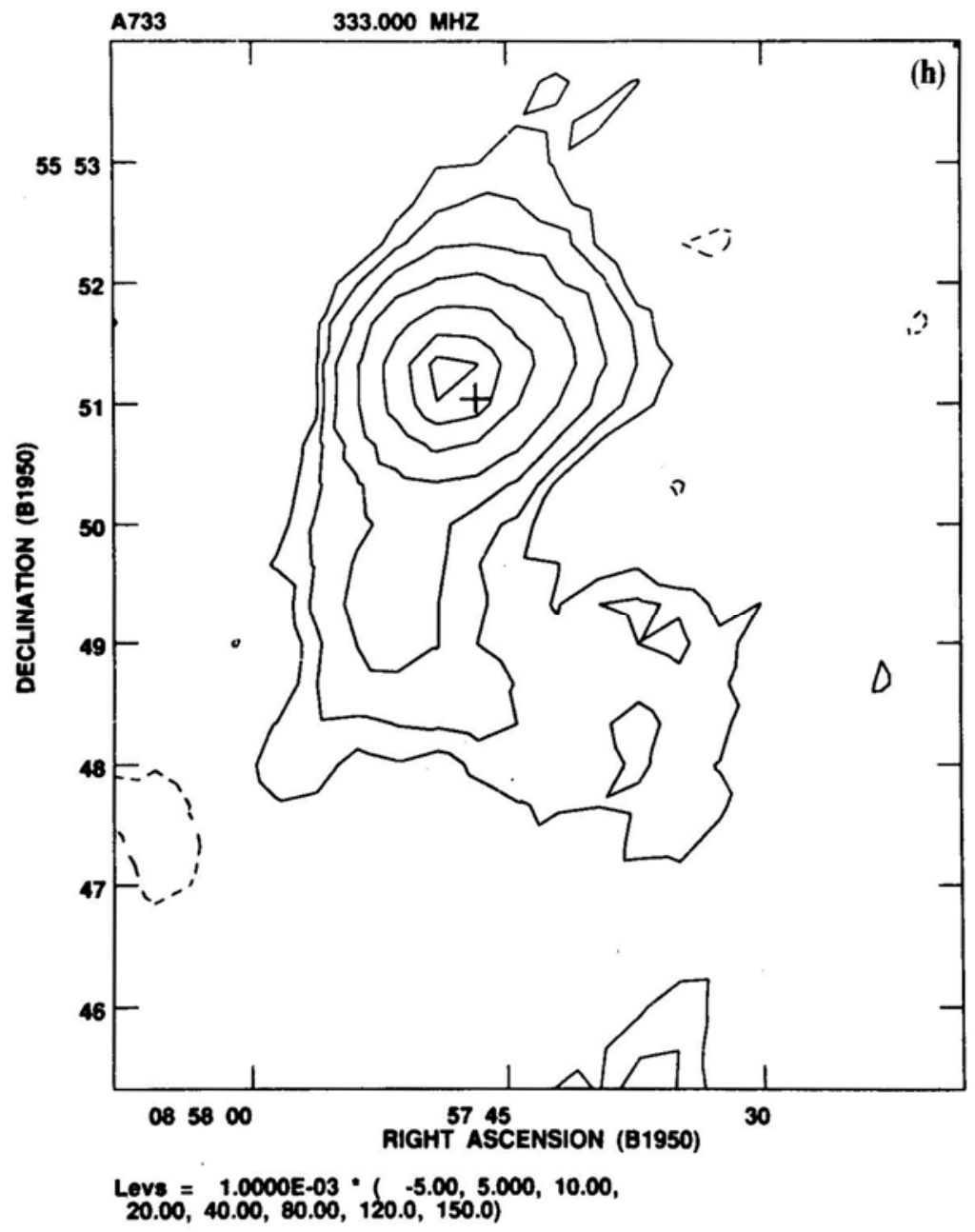

Figure 1 (h).

Now assuming that the bivariate nature of IRLF of brightest radio galaxies is expressible as,

$$
\log \left(F>P_{1.4}\right) \propto M_{p}
$$

we derive, from a regression analysis of Auriemma et al. (1977) data, the following relationships,

$$
\begin{gathered}
\log \left(F>P_{1.4}\right)=-18.12-0.765\left(M_{p}\right) \text { at } \log P_{1.4}=25.0 \text { and }, \\
\log \left(F>P_{1.4}\right)=-14.83-0.556\left(M_{p}\right) \text { at } \log P_{1.4}=25.8 .
\end{gathered}
$$

Both these equations have regression coefficient $r>98 \%$. Using these regression relations and using the mean visual magnitude $\left\langle M_{p}\right\rangle_{\mathrm{cD}}$ as calculated above (with an uncertainty of 0.25 magnitudes), we obtain the following estimates of 


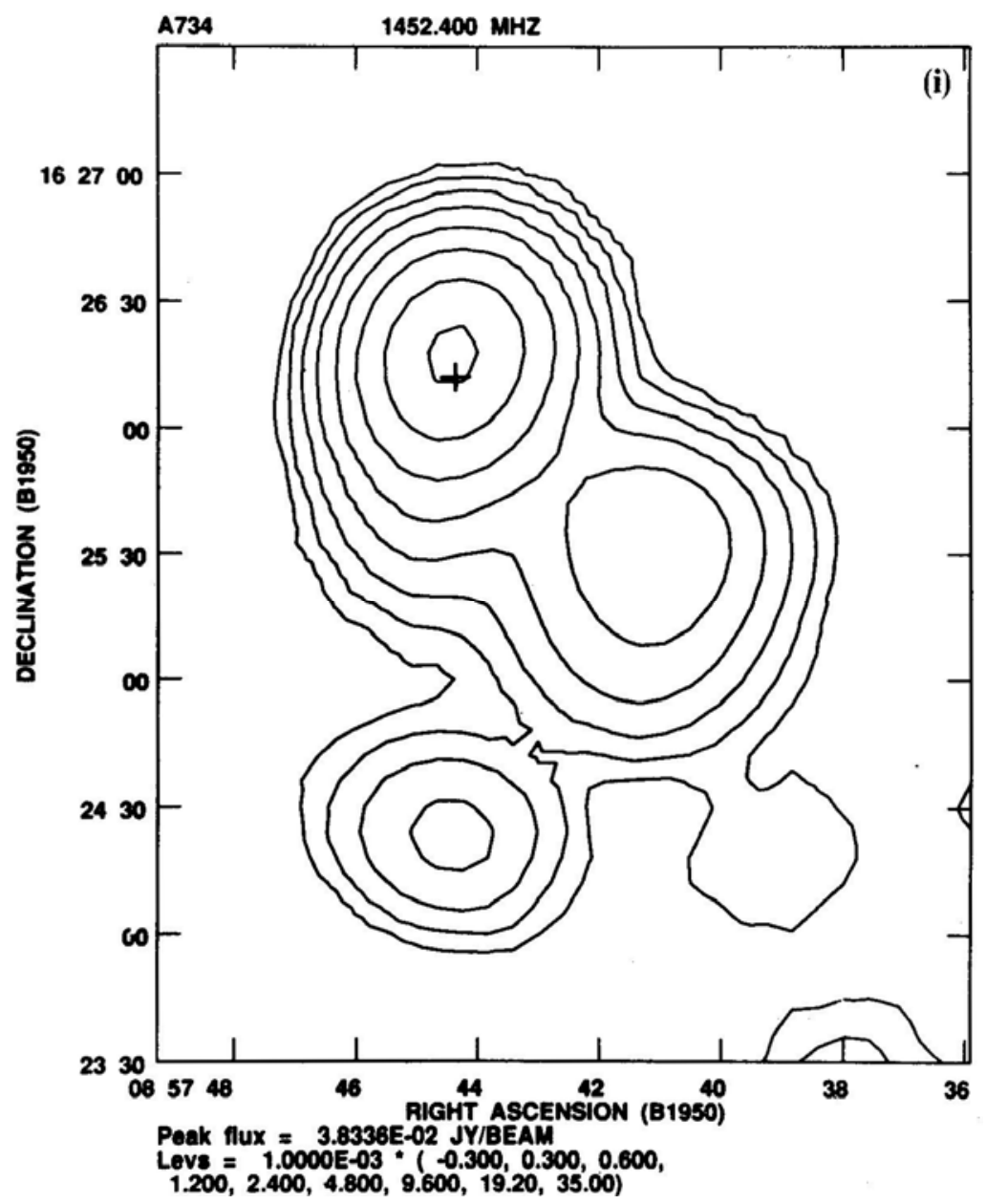

Figure 1 (i)

Probabilities for $\mathrm{cD}$ galaxies.

$$
\begin{gathered}
\left(F>P_{1.4}\right)=0.138\left(\frac{+0.076}{-0.050}\right), \text { at } \log P_{1.4}=25.0 \text { and } \\
\left(F>P_{1.4}\right)=0.005\left(\frac{+0.002}{-0.001}\right), \text { at } \log P_{1.4}=25.8 .
\end{gathered}
$$

These predicted numerical values are consistent with the observed probabilities of $0.07 \pm 0.04$, and $<0.024$ at same two values of $P_{1.4}$ (Table 2, Fig. 2(b)). It thus appears that the physical effect that possibly causes the well known radio-optical correlation, also operates in the case of the cD galaxies in rich clusters. In a typical rich Abell cluster, the space density of galaxies at the location of the $\mathrm{cD}$ can be a factor of 100 or more larger compared to the density around field ellipticals (Dressler 1980). Despite this vast difference, it is striking to note that the RLFs behave identically. This in turn implies that instead of a global, large scale environmental effect, some physical factor dependent mainly upon the local conditions near a radio galaxy is more important in generating a powerful radio source. The local space 


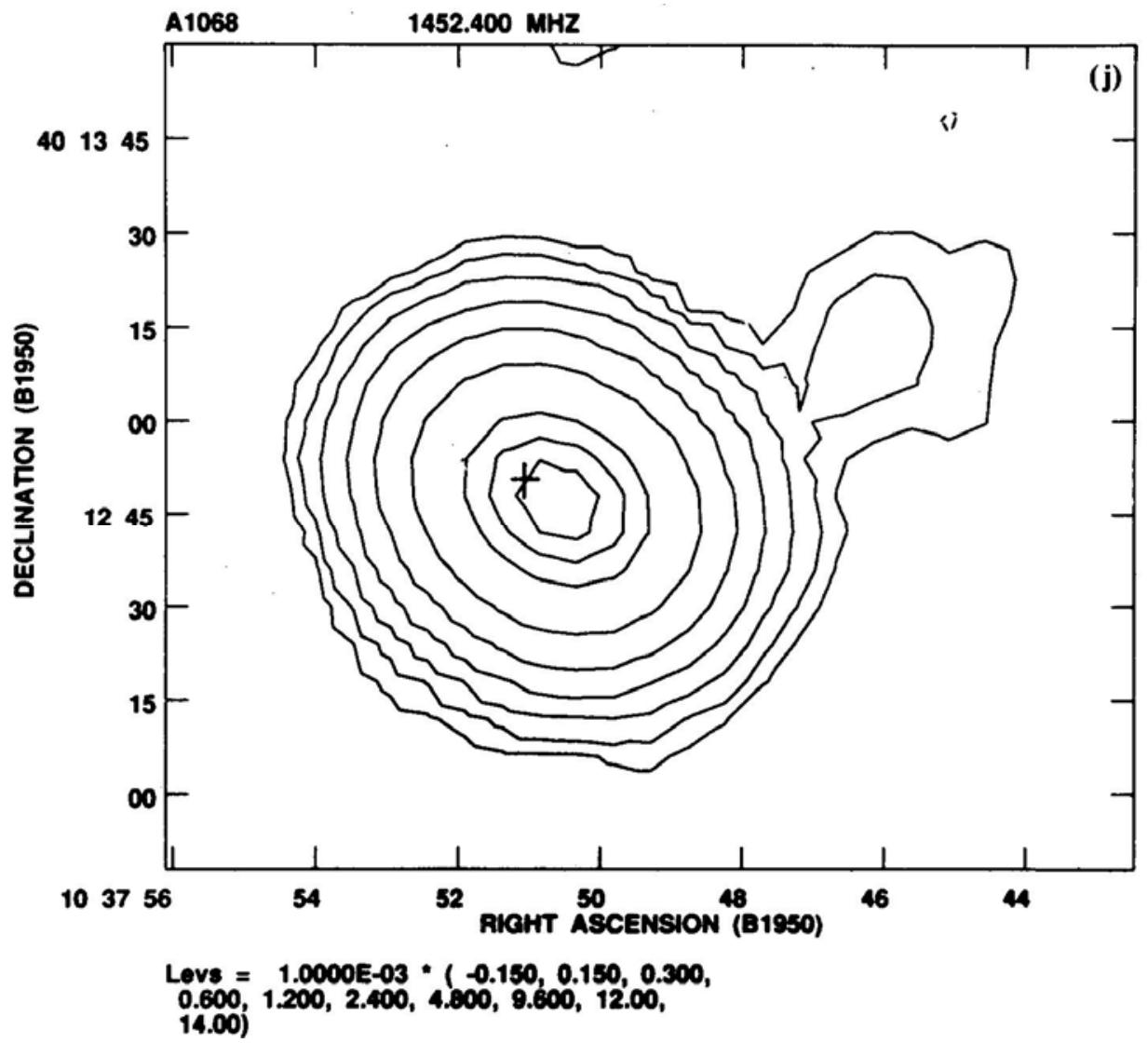

Figure $1(j)$

density of galaxies is unlikely to be this factor because comparison of RLF of ellipticals inside and outside rich clusters fails to indicate any detectable difference (eg. Auriemma et al 1977). Similarly, Valentijn \& Bijleveld (1983) found that the radio luminosity function of $\mathrm{cD}$ galaxies occurring in many poor groups of galaxies is no different from that of elliptical radio galaxies of similar brightness but occurring in sparser regions.

A recent $6 \mathrm{~cm}$ wavelength study of a large number of lower redshift $(z<0.1) \mathrm{cD}$ galaxies also fails to detect any influence of cluster richness on the radio detection probability (Ball et al 1993). This again implies that radio emission probability is more strongly dependent upon the optical brightness of the parent galaxy and less on its global environment. However, it seems clear that $P_{1.4}-\mathrm{Mp}$ correlation is unlikely to be a primary causal relation and is a manifestation of an underlying direct physical relation. The smooth scaling of the probability of radio emission with optical brightness in diverse environments points towards a unified mechanism for triggering of nuclear activity in giant ellipticals and $\mathrm{cD}$ galaxies. Our current understanding of the radio galaxies and quasars indicates that the observed rate of energy production is related to the mass of the central super massive object and to the rate at which gas can be fed to the potential well (Begelman et al. 1984). Thus, it is possible that the more massive and optically brighter galaxies like cDs that form only in clusters, 


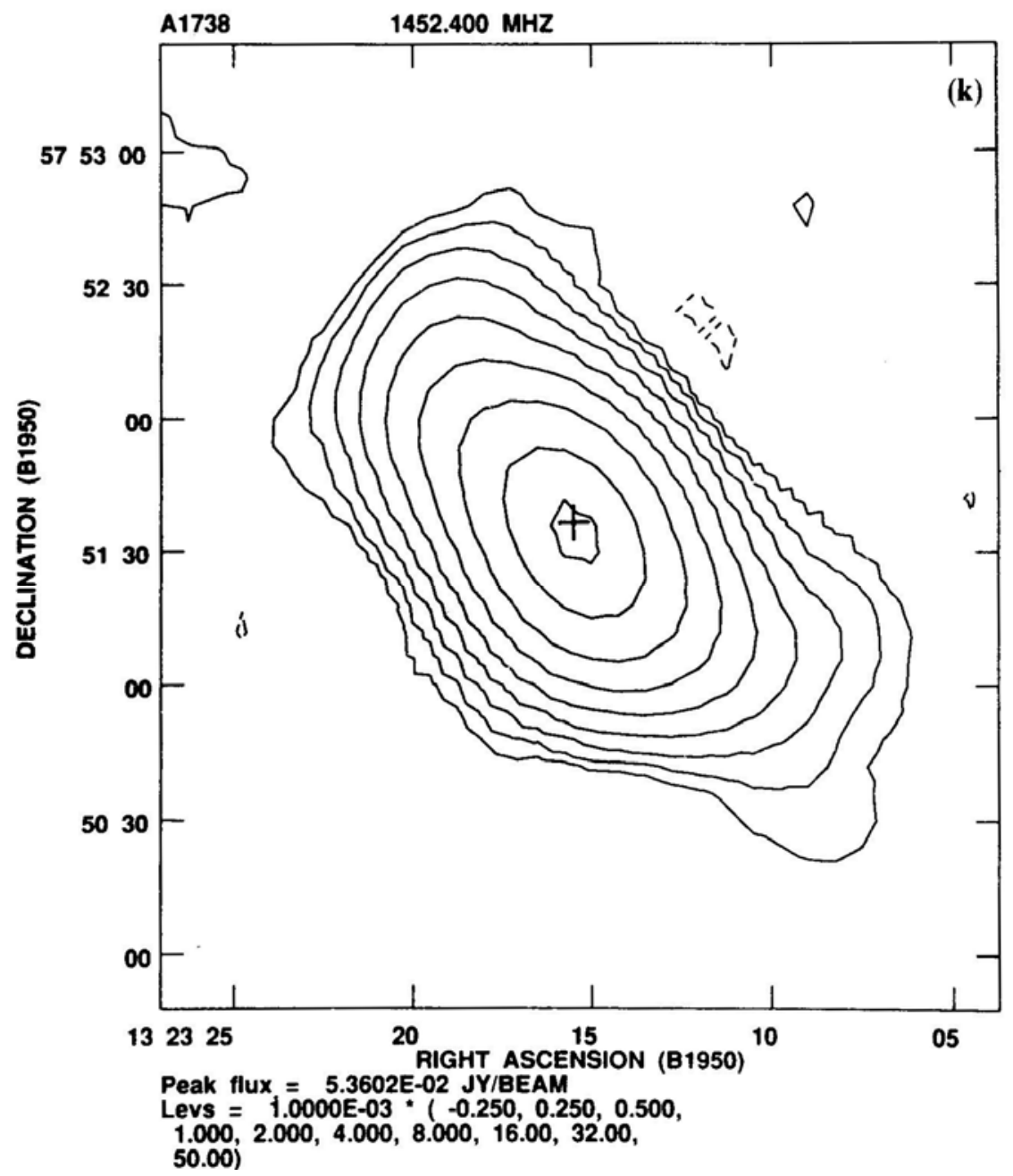

Figure 1 (k).

either harbour more massive central objects or have more efficient fuelling (or both of these), and can thus generate more powerful radio emission. It would be most informative if using high resolution spectroscopic methods, estimates of the masses of the central nuclear regions of these galaxies could be obtained. Some more important clues to the problem can be obtained from the direct study of the fuel for the activity i.e. the gaseous media within and surrounding the radio galaxies. A more detailed study directed towards this aspect is presented in the next section.

\section{Radio emission from $\mathrm{CD}$ galaxies and cooling flows}

As mentioned in the introduction, a strong cosmological evolution in the properties of cosmic radio sources is indicated by observations. It is possible that such effects arise due to dynamical evolution of groups and clusters where such sources are found. 


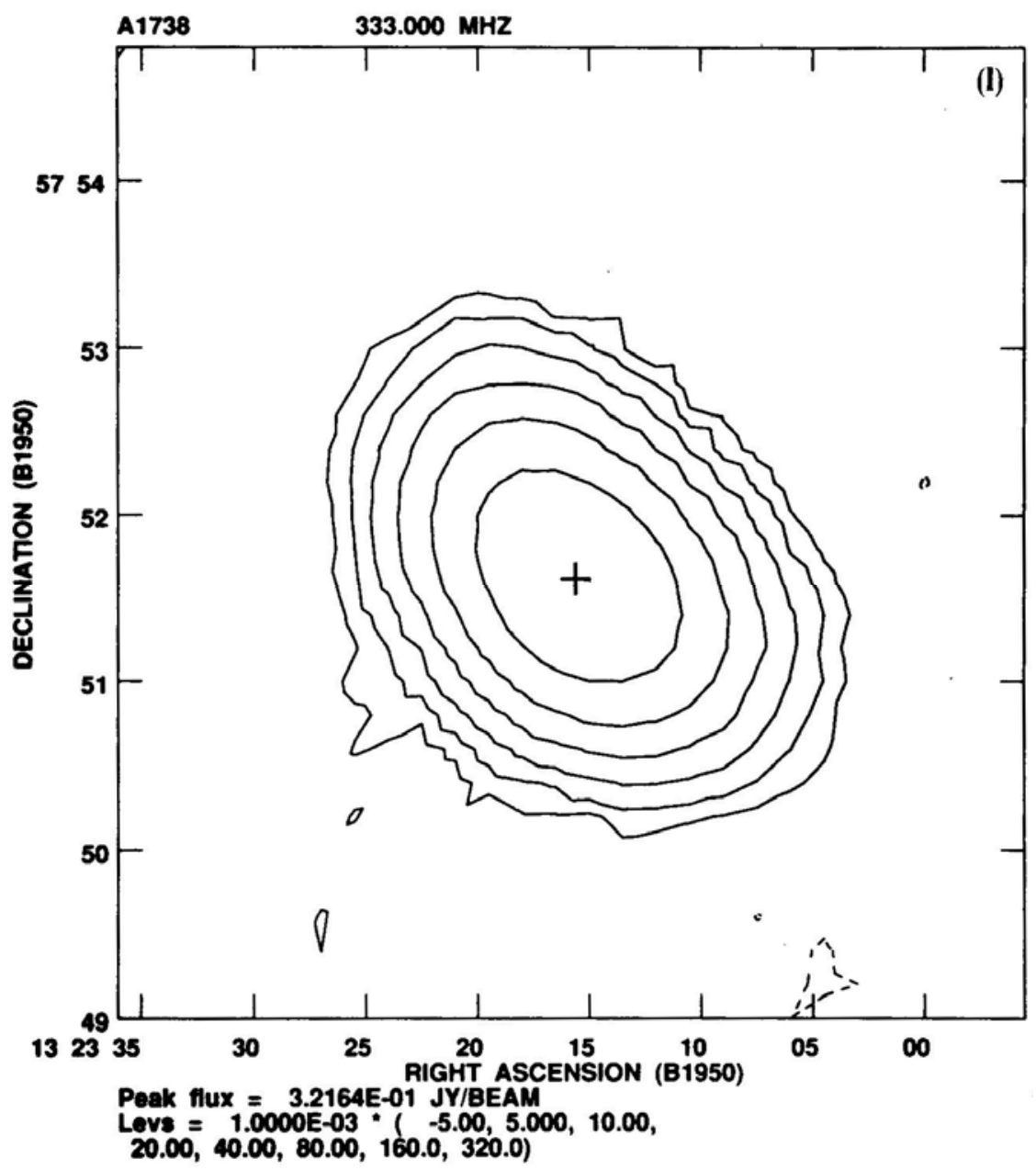

Figure 1 (l).

The quasi-steady flow of cooling gas (inferred from X-ray observations eg. Edge et al. 1992) towards cluster centres is a direct proof of the evolving intracluster medium (ICM). cD galaxies are invariably found at the 'focus' of all such 'cooling-flows' (Fabian 1987, and references therein). One of the models of active galactic nuclei invokes the accretion of gaseous medium as fuel for the nuclear source (Begelman et al. 1984). It is therefore of interest to investigate this possibility. Here we investigate the influence of the physical state of the ICM on radio emission from the central $\mathrm{cD}$ galaxies. Previously, a tendency for the occurrence of strong radio sources in X-ray luminous clusters has been noticed by Valentijn \& Bijleveld (1983), and Burns (1990) has reported that $\mathrm{cD}$ radio sources are more probable in clusters with possible cooling flows. As yet no detailed X-ray data are available for the distant Abell clusters forming our sample. However, the radio and X-ray data already existing for the low- $z \mathrm{cD}$ clusters can provide a useful model of physical processes against which we can compare our radio results. Thus, from published literature we have formed a sample of clusters for which high sensitivity X-ray and radio data (mostly available at $4.8 \mathrm{GHz}$ ) are 


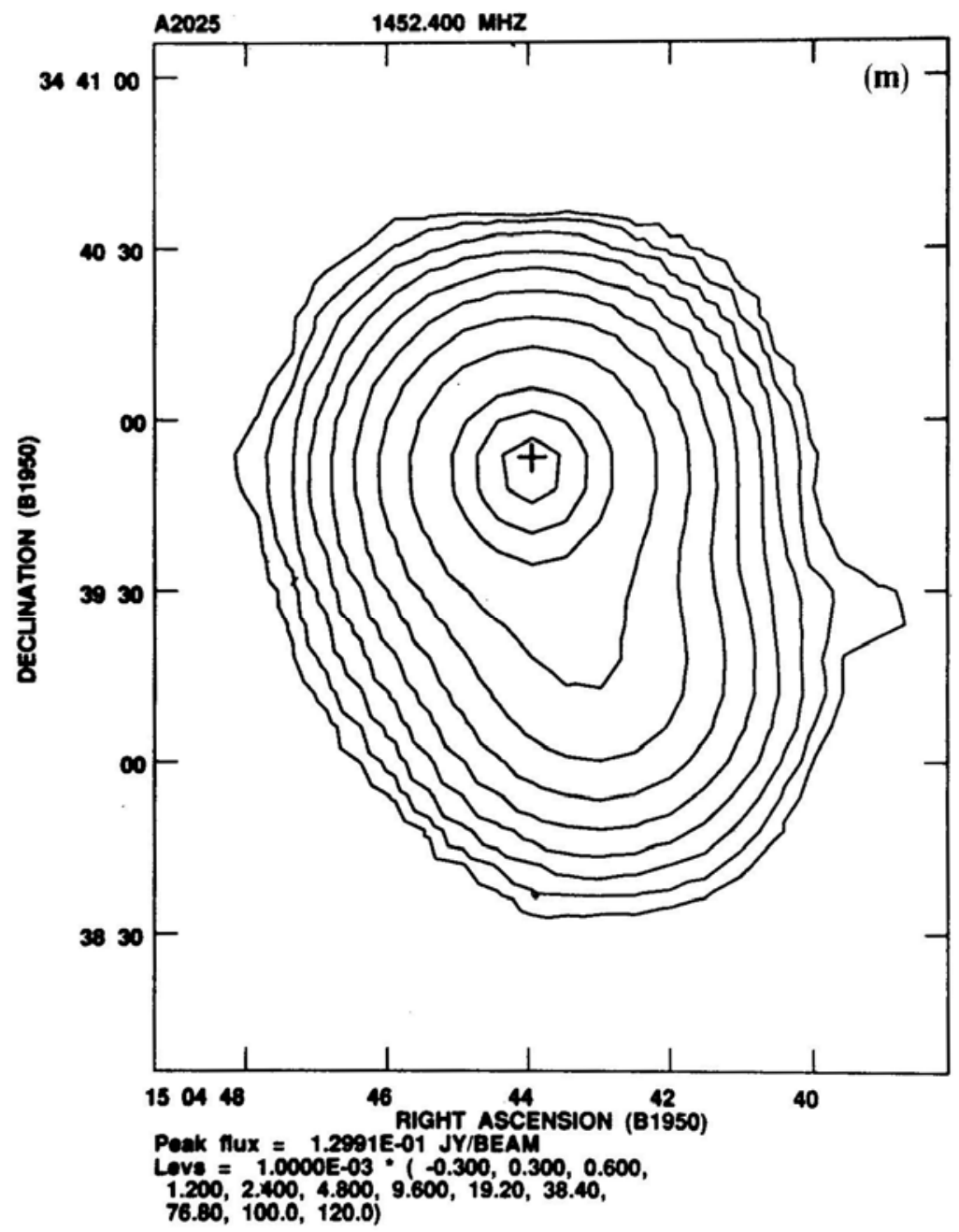

Figure $1(\mathbf{m})$.

available. The main body of data comes from Burns (1990). The radio and X-ray data for cD galaxy PKS 2345-35 is from Schwartz et al. (1991) and radio data on the $\mathrm{cD}$ galaxy $3 \mathrm{C} 318.1$ in the poor cluster MKW3S is from our VLA observations of very steep spectrum radio sources (under preparation), and the X-ray data is from Kriss et al. (1983). The derived physical quantities pertaining to these clusters (cooling time, gas pressure etc.) are again taken from above references. Table 3 summarises this data which is organised as follows.

Col. 1: The cluster designation.

Col.2: The ratio of cooling time $\left(t_{\text {cool }}\right)$ to Hubble time $\left(t_{\text {Hubble }}=\mathrm{H}_{0}^{-1}\right)$ for the ICM in the cluster core.

Col. 3: The redshift of the cluster.

Col. 4: The $4.8 \mathrm{GHz}$ radio luminosity of the $\mathrm{cD}$ galaxy or an upper limit.

Col. 5: The radio spectral index $(\alpha)$ of the $\mathrm{cD}$ between $4.8 \mathrm{GHz}$ and $1.4 \mathrm{GHz}$ (when available); here flux density $\propto$ (frequency $)^{-\alpha}$. 


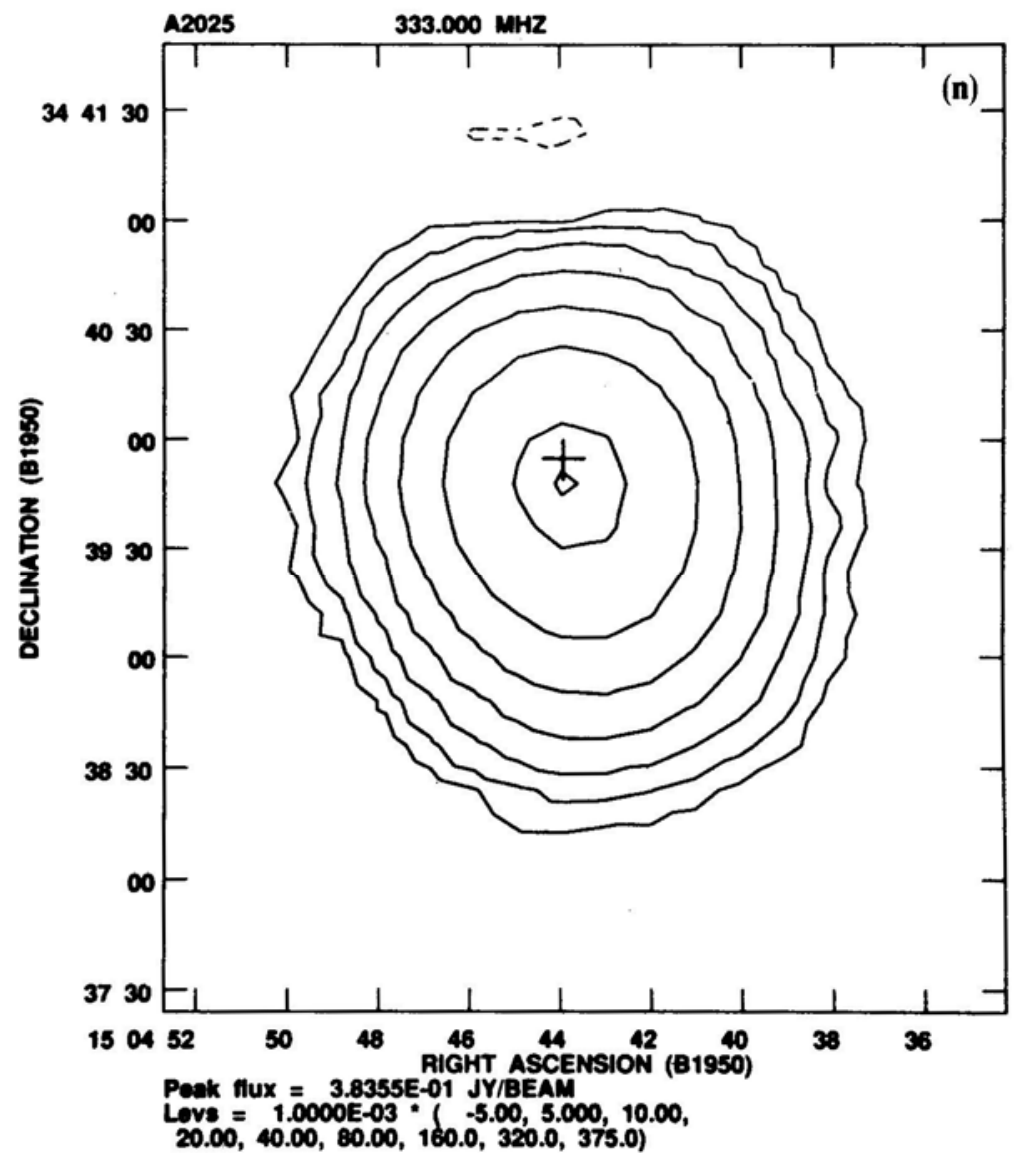

Figure 1 (n).

Col. 6: A code whether a cooling-flow exists $\left(\mathrm{Y}\right.$, if $\left.\frac{t_{\text {cool }}}{t_{\text {Hubble }}}<1\right)$ or not

$$
\left(\mathrm{N}, \text { if } \frac{t_{\text {cool }}}{t_{\text {Hubble }}} \geqslant 1\right) \text {. }
$$

Col. 7: The gas pressure of the ICM in the cluster core.

It is seen from Table 3 that the cooling time at the centres of many clusters is less than the Hubble time $\left(t_{\text {cool }}<t_{\text {Hubble }}>\right.$ where $t_{\text {Hubble }} \approx 2 \times 10^{10} \mathrm{yr}$ for $H_{0}=50 \mathrm{~km}$ $\left.\mathrm{sec}^{-1} \mathrm{Mpc}^{-1}\right)$. These are the clusters in which there is a strong possibility of finding a cooling-flow in operation. The implied mass inflow rate generally is in the range of $\left(10 M_{\odot}-500 M_{\odot}\right) \mathrm{y}^{-1}$ (Fabian 1987). Can a massive flow of cool gas towards the cluster core influence the radio emission from $\mathrm{cD}$ and other galaxies? To investigate this, we plot the $4.8 \mathrm{GHz}$ radio luminosity of $\mathrm{cDs}\left(\mathrm{P}_{4.8}\right.$ in $\left.\mathrm{WHz}^{-1}\right)$ versus the normalised cooling time $\left(\frac{t_{\text {cool }}}{t_{\text {Hubble }}}\right)$ in Fig. 3. Clearly, despite a substantial scatter, There is an anti- correlation between $\log \left(P_{4.8}\right)$ and $\log \left(\frac{t_{\text {cool }}}{t_{\text {Hubble }}}\right)$. A least square 


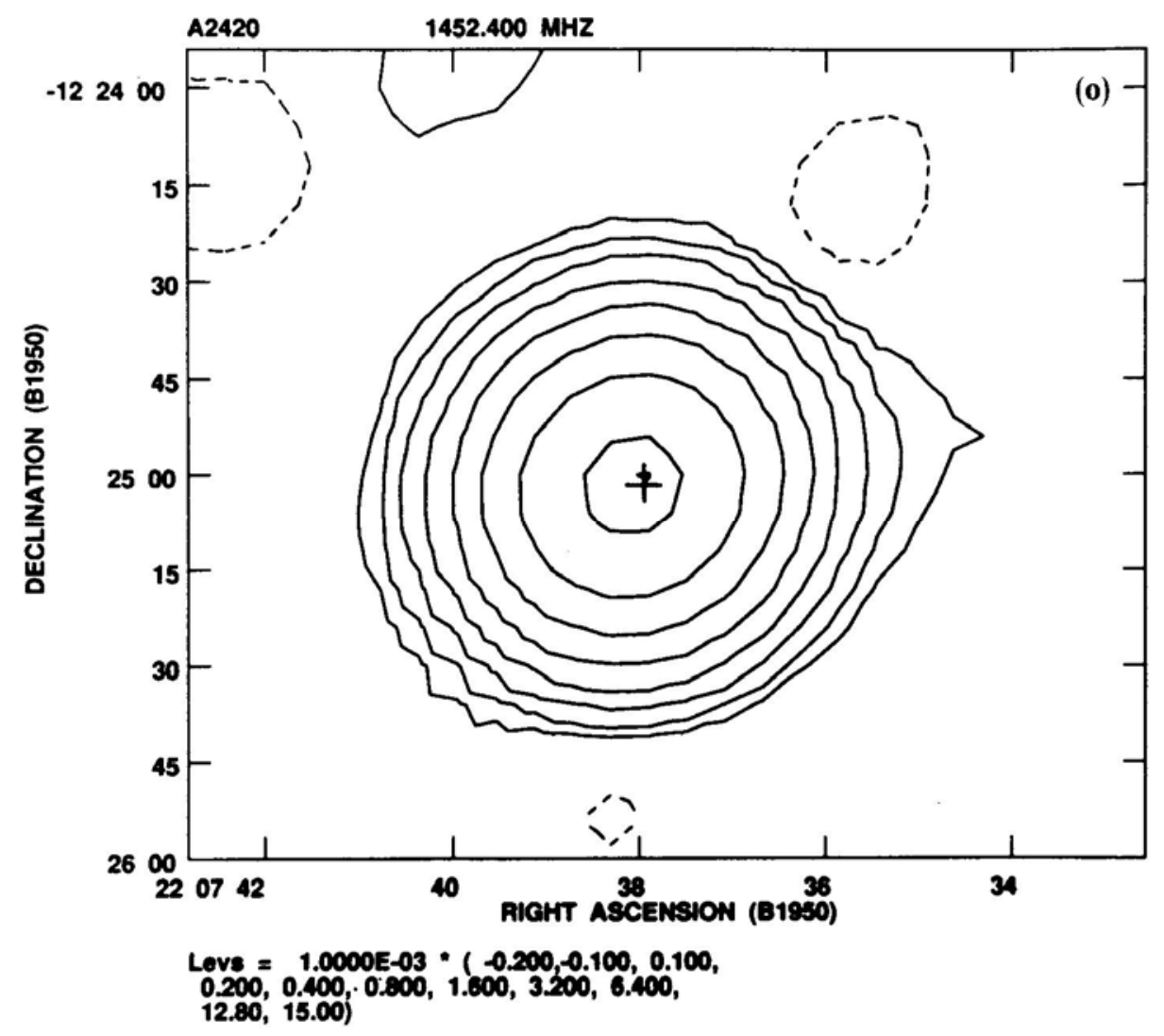

Figure 1 (o).

Figure 1 a-o. Radio maps of 11 resolved sources (LAS $\gtrsim 10 \operatorname{arcsec}$ ) at $1.4 \mathrm{GHz}$ and 4 sources also resolved at $330 \mathrm{MHz}$. The FWHM sizes of the CLEAN beams are $30 \operatorname{arcsec} \times 30 \operatorname{arcsec}$ at $1.4 \mathrm{GHz}$ and $60 \operatorname{arcsec} \times 60 \operatorname{arcsec}$ at $330 \mathrm{MHz}$. The optical position of the cD galaxy is marked by a + symbol and each map contains the cluster name at upper left corner. The peak of the flux density and the contour levels in Jy units are indicated at the bottom. The peak flux density in some maps (not listed at the bottom), in units of $\mathrm{mJy} /$ beam is as follows:

A22:20.0mJy/beam; A360:8.4; A394:12.3; A690(0.33GHz): 189.7; A733(1.4GHz):28.8, (0.33 GHz) 159.6; A1068:15.4; A2420:15.1.

regression analysis of the data yields the following relation having a regression coefficient $r=0.78$ (including the upper limits).

$$
\log \left(P_{4.8}\right)=22.31-1.69 \times \log \left(\frac{t_{\text {cool }}}{t_{\text {Hubble }}}\right)
$$

It was noted by Owen et al. (1984) that wide-angle tailed (WAT) sources have large angular sizes and are radio luminous but tend to avoid cooling-flow clusters. The same is true for two WAT sources shown in Fig. 3. Burns (1990) also notes this feature and points out that these sources are morphologically distinct. The physical cause of this effect is not yet known but the mechanism responsible for the origin and the shape of these sources is separate from other $\mathrm{cD}$ radio sources. In view of this uncertainty, we have excluded two WAT sources and confined ourselves to the 
Table 2. Differential and integral RLF of $\mathrm{cD}$ galaxies.

\begin{tabular}{lcccccc}
\hline $\begin{array}{l}\log \left(P_{1.4}\right) \\
(\text { Watt/Hz) }\end{array}$ & $\begin{array}{l}\text { Number } \\
\text { surveyed }\end{array}$ & $\begin{array}{l}\text { Number } \\
\text { detected }\end{array}$ & DRLF & (error) & IRLF & (error) \\
\hline $21.8-22.2$ & 01 & 0 & - & - & 0.438 & $(0.113)$ \\
$22.2-22.6$ & 03 & 0 & - & - & 0.438 & $(0.113)$ \\
$22.6-23.0$ & 22 & 2 & 0.091 & $(0.064)$ & 0.438 & $(0.113)$ \\
$23.0-23.4$ & 33 & 1 & 0.030 & $(0.030)$ & 0.347 & $(0.093)$ \\
$23.4-23.8$ & 41 & 3 & 0.073 & $(0.042)$ & 0.317 & $(0.088)$ \\
$23.8-24.2$ & 41 & 1 & 0.024 & $(0.024)$ & 0.244 & $(0.077)$ \\
$24.2-24.6$ & 41 & 5 & 0.122 & $(0.055)$ & 0.220 & $(0.073)$ \\
$24.6-25.0$ & 41 & 2 & 0.049 & $(0.034)$ & 0.098 & $(0.048)$ \\
$25.0-25.4$ & 41 & 2 & 0.049 & $(0.034)$ & 0.049 & $(0.034)$ \\
$25.4-25.8$ & 41 & 0 & $<0.024$ & & $<0.024$ & \\
\hline
\end{tabular}

Arrangement of Columns:

1. Log radio luminosity at $1.4 \mathrm{GHz}$ in $\mathrm{Watt} / \mathrm{Hz}$.

2. Number of clusters surveyed.

3. Number of clusters detected.

4. Differential RLF with errors in brackets.

5. Integral RLF with errors in brackets.

'average' cD radio sources. From equation 4 above, it is implied that $\mathrm{cD}$ radio sources with $\log \left(P_{4 \cdot 8}\right) \gtrsim 22.31 \mathrm{WHz}^{-1}$ occur more often $(\approx 65 \pm 20 \%)$ in clusters with $\left(\frac{t_{\text {cool }}}{t_{\text {Hubble }}}\right)<1$, or those with cooling flows. The corresponding value of this radio luminosity at $1.4 \mathrm{GHz}$ is $\log \left(P_{1.4}\right)=22.7 \mathrm{WHz}^{-1}$ for a spectral index $\alpha=0.75$. This is just about the weakest radio luminosity that can be detected in our more distant $(z>0.1)$ sample of $\mathrm{cD}$ clusters. From the derived RLF of these cDs (see Table 2, Fig. 2), we expect $\approx 40 \% \pm 10 \%$ of galaxies to have associated radio sources with $\log$ $\left(P_{1.4}\right)>22.7 \mathrm{WHz}^{-1}$. Thus, it appears that the probability of radio emission from cDs in cooling flows is slightly higher than that of average cluster cDs. Valentijn \& Bijleveld (1983) also present evidence for enhanced probability of radio emission from cDs in X-ray bright (an indirect evidence for a probable cooling flow) clusters of galaxies. The association of radio sources occurring in $\mathrm{SO}, \mathrm{E}$ and $\mathrm{cD}$ galaxies with the cooling time of the ICM was investigated by Zhao et al. (1989) but they failed to find any significant correlation. It is quite likely that the cooling time versus radio power correlation holds only for $\mathrm{cD}$ galaxies which occupy a unique central position in cooling flow systems. Thus, we conclude that the physical state of the intracluster medium influences the probability of radio emission from $\mathrm{cD}$ galaxies. Powerful radio sources appear more likely to originate in clusters with cooling cores and the radio luminosity correlates inversely with the cooling time of the ICM. Two possible physical causes capable of producing such a correlation are: (i) the dense gas of a cooling-flow surrounding a cD galaxy leads to an efficient confinement of the radio plasma, rendering it visible due to prevention of adiabatic expansion losses; (ii) a fraction of the cool gas is accreted by the $\mathrm{cD}$ galaxy which then fuels the central engine of its active nucleus. 

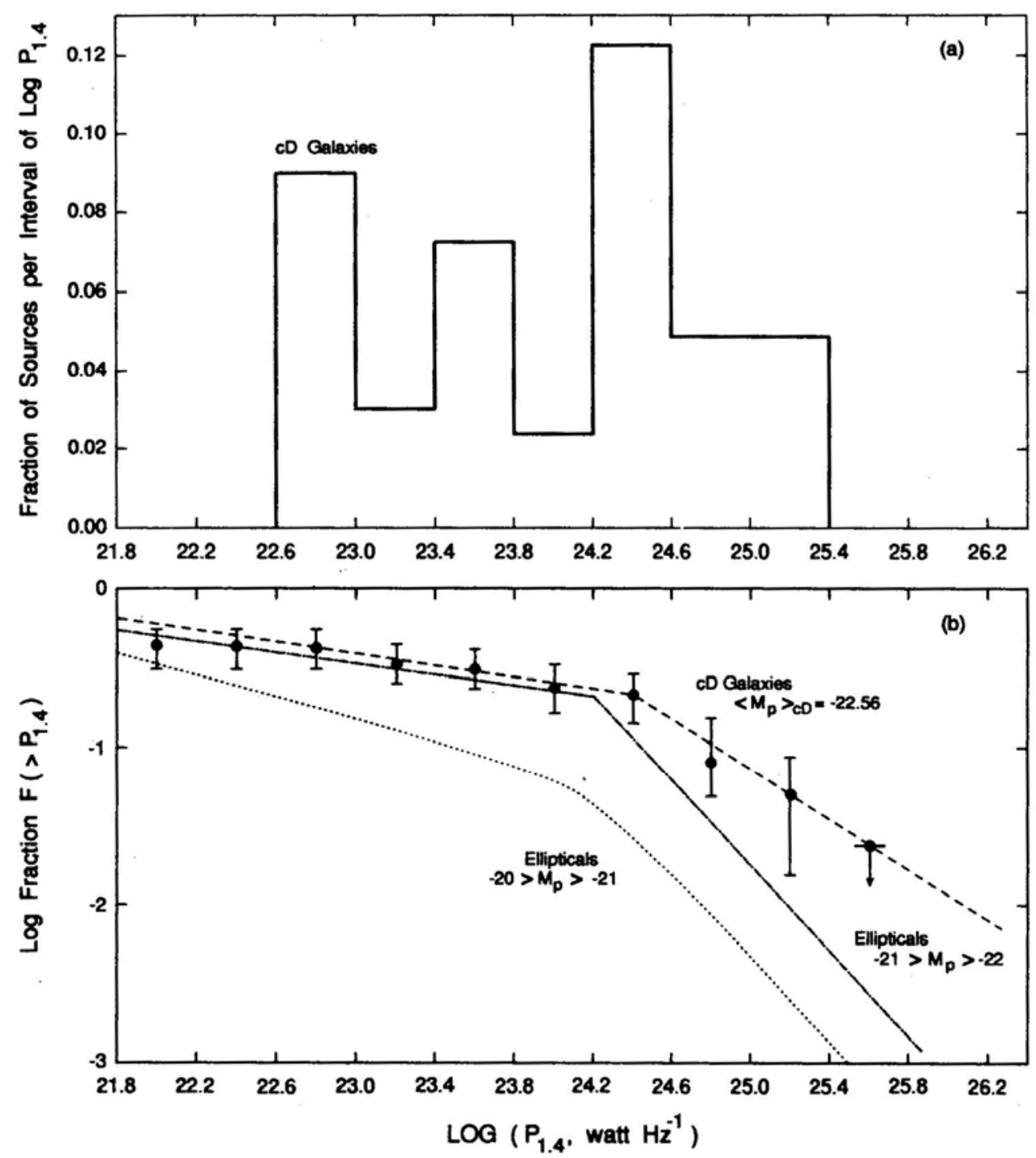

Figure 2. The differential (a) and integral (b) radio luminosity function for $\mathrm{cD}$ galaxies at $1.4 \mathrm{GHz}$. In Fig. 2(b) the dashed curve shows the best fitting function for the cD galaxies and the dot-dashed and dotted curves represent the functions for giant elliptical galaxies in the photographic magnitude ranges $(-22,-21)$ and $(-21,-20)$ obtained from the data of Auriemma et al. (1977).

We note that both the confinement and fuelling of a $\mathrm{cD}$ radio source can happen simultaneously by the ICM. The gas pressure of the ICM, $P_{\text {gas }}=n_{e} \mathrm{k} T_{g}$ for electron density $n_{e}$ and temperature $T_{g}$. The data on $P_{\text {gas }}$ in Table 3 show that the average $\mathrm{P}_{\text {gas }}$ for cooling flow clusters is $\approx 4 \times 10^{-11} \mathrm{dyn} \mathrm{cm}^{-2}$ as against $\approx 3 \times 10^{-11}$ dyn$\mathrm{cm}^{-2}$ for non-cooling flow ones. Thus, given the uncertainties, the gas pressure is not much different in two types of clusters and is capable of confining all but the most luminous and compact of radio sources. This implies that thermal confinement 
Table 3. Data on cD clusters with X-ray emission.

\begin{tabular}{|c|c|c|c|c|c|c|}
\hline Cluster & $\frac{t_{\text {cool }}}{t_{\text {Hubble }}}$ & $\begin{array}{c}\text { Redshift } \\
(z)\end{array}$ & $\begin{array}{c}\text { Log radio } \\
\text { lumin. } \\
P_{6}\left(\mathrm{~W} \mathrm{~Hz}^{-1}\right)\end{array}$ & $\begin{array}{c}\text { Spectral } \\
\text { index } \\
\alpha\end{array}$ & $\begin{array}{l}\text { Cooling } \\
\text { flow } \\
(\mathrm{Y} / \mathrm{N})\end{array}$ & $\begin{array}{c}\text { Gas pressure } \\
\times 10^{-11} \\
\text { dyn }-\mathrm{cm}^{-2}\end{array}$ \\
\hline $0745-191$ & 0.1 & 0.1028 & 25.25 & 1.36 & $\mathrm{Y}$ & 19.3 \\
\hline MKW3S & 0.4 & 0.0434 & 22.28 & $<0.83$ & $\mathrm{Y}$ & 1.1 \\
\hline PKS $2345-35$ & 0.2 & 0.0480 & 23.99 & - & $\mathrm{Y}$ & 6.7 \\
\hline A85 & 0.4 & 0.0518 & 23.13 & 1.08 & $\mathrm{Y}$ & 5.1 \\
\hline A133 & 0.4 & 0.0570 & 22.99 & 2.82 & $\mathrm{Y}$ & 1.9 \\
\hline A399 & 4.8 & 0.0715 & $<21.77$ & - & $\mathrm{N}$ & 5.0 \\
\hline A401 & 1.0 & 0.0748 & $<22.05$ & - & $\mathrm{N}$ & 3.9 \\
\hline A496 & 0.1 & 0.0316 & 23.34 & 0.20 & $\mathrm{Y}$ & 1.4 \\
\hline A644 & 0.5 & 0.0781 & $<21.78$ & - & $\mathrm{Y}$ & 2.4 \\
\hline A671 & 1.7 & 0.0497 & $<21.35$ & - & $\mathrm{N}$ & 1.0 \\
\hline A 1650 & 2.7 & 0.140 & $<22.40$ & - & $\mathrm{N}$ & 6.1 \\
\hline A1767 & 0.7 & 0.0756 & $<21.74$ & - & $\mathrm{Y}$ & 1.2 \\
\hline A1795 & 0.2 & 0.0621 & 24.59 & 1.07 & $\mathrm{Y}$ & 4.5 \\
\hline A1809 & 3.2 & 0.0788 & $<21.90$ & - & $\mathbf{N}$ & 1.4 \\
\hline A 1890 & $>1.0$ & 0.0580 & $<21.59$ & - & $\mathrm{N}$ & 1.0 \\
\hline A2029 & 0.3 & 0.0767 & 24.38 & 1.31 & $\mathrm{Y}$ & 4.7 \\
\hline A2052 & 0.1 & 0.0345 & 24.68 & 1.23 & $\mathrm{Y}$ & 1.2 \\
\hline A2063 & 0.2 & 0.0337 & 21.86 & 2.28 & $\mathrm{Y}$ & 1.3 \\
\hline A2107 & 1.9 & 0.0421 & $<21.24$ & - & $\mathbf{N}$ & 2.0 \\
\hline A2124 & 2.0 & 0.0669 & $<21.52$ & - & $\mathrm{N}$ & 0.9 \\
\hline A2199 & 0.2 & 0.0305 & 24.32 & 1.35 & $\mathrm{Y}$ & 3.5 \\
\hline A2244 & 0.6 & 0.0997 & $<21.82$ & - & $\mathrm{Y}$ & 3.6 \\
\hline A2271 & 8.3 & 0.0616 & $<21.48$ & - & $\mathrm{N}$ & 1.4 \\
\hline A2319 & 1.8 & 0.0529 & $<21.41$ & - & $\mathrm{N}$ & 9.5 \\
\hline A2420 & 2.0 & 0.0838 & 23.21 & - & $\mathrm{N}$ & 2.5 \\
\hline A2593 & 2.8 & 0.0440 & $<21.20$ & - & $\mathrm{N}$ & 0.8 \\
\hline A2626 & 0.8 & 0.0562 & 23.03 & 1.37 & $\mathrm{Y}$ & 1.6 \\
\hline A2634 & 2.3 & 0.0322 & 25.10 & 0.70 & $\mathbf{N}$ & 0.6 \\
\hline A2670 & 2.0 & 0.0749 & 22.71 & - & $\mathrm{N}$ & 2.8 \\
\hline
\end{tabular}

is unlikely to be a sufficient factor. However, confinement is necessary or else the lifetime of sources would be « $10^{8}$ years due to adiabatic expansion losses. The fairly steep spectral indices $(\alpha \gtrsim 1.0)$ for the cases where these are measured (Table 3$)$ also indicate efficient confinement. In section 8 a more detailed analysis of observable effects of confinement is presented.

A definite proof for fuelling is difficult to obtain because once the gas cools below the X-ray emission temperature $\sim 10^{6} \mathrm{~K}$, its fate is not clearly known (Fabian et al. 1984, 1991). The gas can eventually get accreted by the central galaxy as its virial temperature $\left(\sim 10^{7} \mathrm{~K}\right)$ is of the same order as the cold gas of a cooling flow. As little as a few $M_{\odot} \mathrm{y}^{-1}$ of gas supply is enough to fuel the active nuclei of most powerful radio sources. In order to accrete efficiently, the speed of the galaxy relative to cluster medium must be small ( $\lesssim 100 \mathrm{~km} \mathrm{sec}^{-1}$ ) and thus cluster members located near the deepest regions of potential wells would be efficient accretors. The location of the $\mathrm{cD}$ galaxies and other giant ellipticals near the centre of cluster potential wells (Dressier 1980), and the observation that radio sources are also found most often 


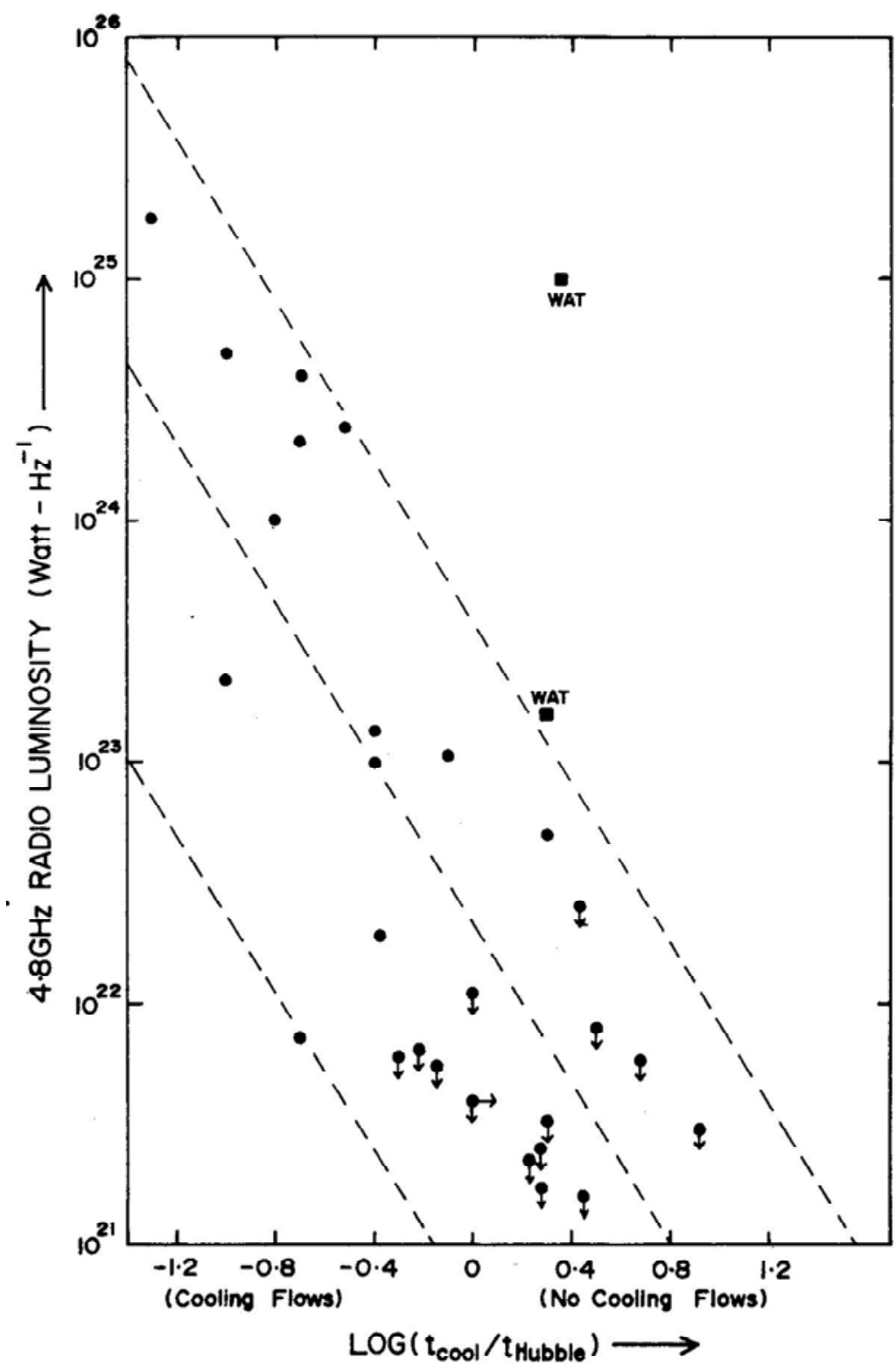

Figure 3. Plot of the radio luminosity of $\mathrm{cD}$ galaxies versus cooling time of the intracluster gas in the clusters. Radio luminosity at $4.8 \mathrm{GHz}$ (in $\mathrm{WHz}^{-1}$ ) is plotted against the log normalized cooling time $\left(t_{\text {cool }} / t_{\text {Hubbie }}\right)$ for the intracluster medium in the cluster core. Upper limits on radio luminosities are indicated by arrows. The central dashed line is the best fitting regression line described in the text and the other two dashed lines represent the upper and lower envelopes. Two wide-angled-tailed (WAT) radio sources are shown by a filled square symbol.

near the cluster centres (McHardy 1979), are strong supportive evidences for the accretion hypothesis.

Optical observations of radio loud quasars indicate that their host galaxies havelight profiles of giant elliptical galaxies (eg. Hutchings et al. 1981) and for $z \gtrsim 0.5$, 
they tend to lie in clusters of richness comparable to Abell clusters (Yee \& Green 1987). The presence of massive cooling flows in the extranuclear regions of many of these quasars is indicated by the detection of extended emission line gas in pressure equilibrium with the external ICM (Crawford et al. 1988; Crawford \& Fabian 1989). It has been suggested that the sudden fading of radio loud quasars since $z \lesssim 0.5$ is related to the disruption of gas supply available from massive cooling flow systems during the merger of subunits containing such quasars (Fabian \& Crawford 1990). The final end products of such evolution in clusters are the massive $\mathrm{cD}$ like galaxies lying at the centres of more dynamically evolved clusters. Thus, the presence of massive cooling flows in low redshift clusters and the evidence of a correlation between the radio luminosity and cooling time strongly suggest that due to the revival of cooling flows in dynamically evolved clusters, the radio emission from the central regions of massive galaxies is also being revived. The evidence presently available to us is supportive of the accretion hypothesis. It would be quite useful if the link between the radio emission from central galaxies in clusters and the cooling flows could be further investigated by the radio and X-ray wavelength study of a larger sample of clusters spanning a wider range in redshift. A recent X-ray observation in this direction indicates a factor $\sim 2$ decline in number of cooling flows since $z=0.3$, presumably due to subcluster mergers (Donahue et al. 1992). A further investigation of the relationship between the radio emission from $\mathrm{cDs}$ and the cluster dynamics is presented in the next section.

\section{Link between cD galaxy location and radio emission}

In this section we investigate, whether the location of a $\mathrm{cD}$ galaxy with respect to the centre of the cluster has any effect on its radio emission. This question is motivated by the fact that we are attempting to understand the nature of radio emissions from the optically brightest cluster members in rich clusters. X-ray data from the EINSTEIN satellite have already given the important clue that many of the brightest galaxies in clusters are uniquely placed at the centres of gravitational potential wells traced out by the hot intracluster gas (Jones \& Forman 1984). Our study regarding the cooling flows in such clusters, presented in section 6, indicates a strong dependence of radio luminosity upon the cooling time of the intracluster medium, which is indicative of the dynamical state (virialized/irregular) of the central region of the cluster. Thus, it is of interest to study the related question of the effect of the position of the brightest cluster galaxies relative to the geometrical centres of cluster (again indicative of the dynamical state of the cluster), upon the radio emission from such galaxies. The geometrical centres of these clusters have already been determined using visual methods by Abell (1958) and Abell et al. (1989), and we have used these measurements. The range of uncertainty in these measurements is of the order of 1 to 3 arcmin. We divide the cD galaxies with optically measured coordinates (41 in number, Table 1) into two groups:

- Radio Louds (13); those that have been detected as radio sources at $1.4 \mathrm{GHz}$, having a radio luminosity equal or above the limit $P_{\lim }=10^{23.45} \mathrm{WHz}^{-1}$ and,

- Radio Quiets (28); the ones that have their $1.4 \mathrm{GHz}$ radio luminosities below this $P_{\lim }$ 
It needs to be mentioned here that the luminosity threshold $P_{\lim }=10^{23.45} \mathrm{WHz}^{-1}$ corresponds to the minimum detectable radio luminosities from the clusters with largest redshifts $(z \approx 0.25)$ in our sample. This choice of threshold radio luminosity was made in order to avoid a selection bias causing selection of less radio luminous sources from lowz clusters and viceversa. A K-S test shows that the redshift distributions of the cDs in the two groups are statistically indistinguishable. The distribution of the angular distances of the optical positions of the $\mathrm{cD}$ galaxies (r) as measured from their cluster centres is presented in Figs. 4(a) and (b) for radio-quiet and radioloud cDs respectively. The displayed angular distances are normalised with respect to the Abell radius $R_{A}\left(R_{A}=3 \mathrm{Mpc}\right)$. It is seen that the distribution of $r / R_{A}$ for radioquiet $\mathrm{cDs}$ is much wider compared to the radio-louds which are more centrally located. The median value of $r / R_{A}$ for radio-quiet $\mathrm{cDs}$ is found to be $\approx 0.13$ whereas, for the radio-loud group it is only $\approx 0.06$. A Kolmogorov-Smirnov test performed

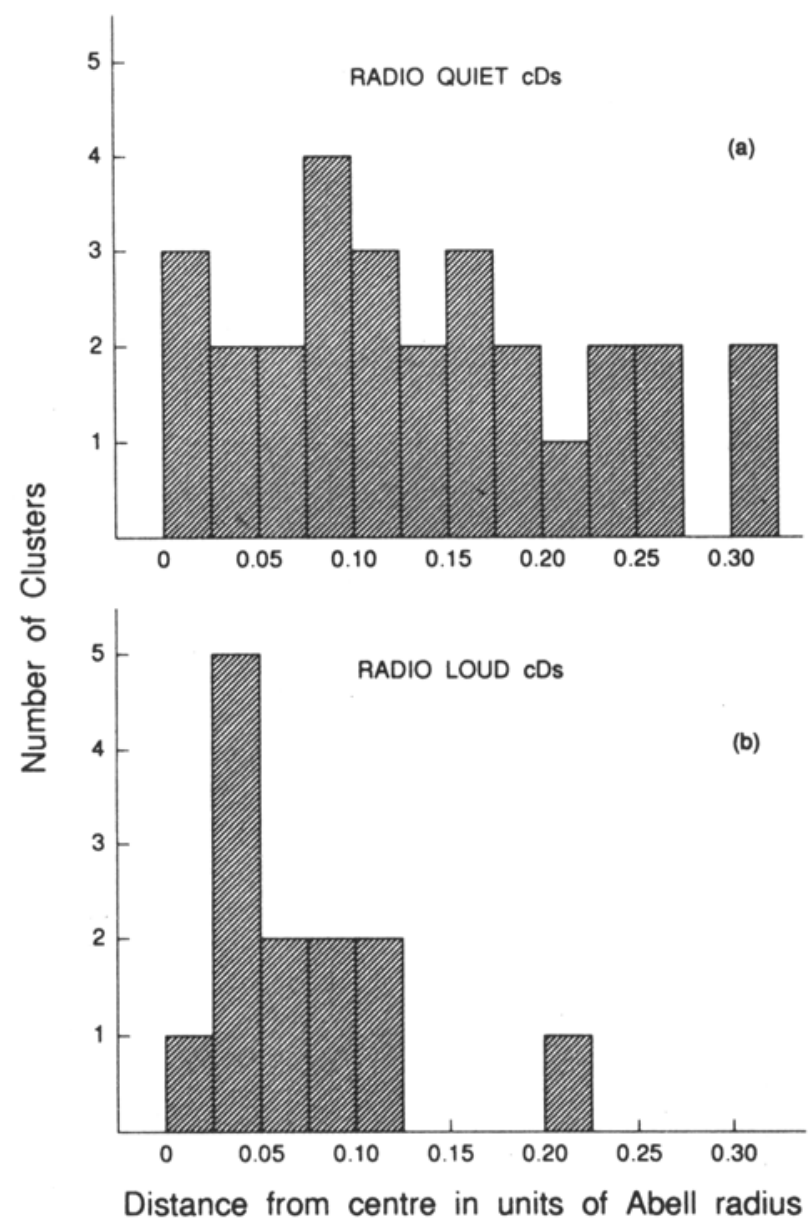

Figure 4. A histogram of the location of radio-quiet (a) and radio-loud (b) cD galaxies in Abell Clusters Plotted are the number of cDs in different radial bins from the cluster centres (in units of Abell radius $R_{A}=3 \mathrm{Mpc}$ ). 
with null-hypothesis (the two distributions are identical) and test-hypothesis (that the radio-quiet sample is more extended), rejects the null hypothesis at a significance level of $\approx 0.03(3 \%)$. Thus, we conclude that the radial distribution of radio-quiet $\mathrm{cD}$ galaxies is different and more extended compared to distribution of radio-loud cDs at $\approx 97 \%$ level of significance. As the cluster centres are determined only to an accuracy of 1 to 3 arcmin or so, the average error in cluster centre positions must $\mathrm{be} \approx 0.16$ in Abell radius units. We find that 12 out of $13(92 \%)$ of the radio-loud $\mathrm{cDs}$ are situated within this range. It is therefore possible that the actual distribution of $r / R_{A}$ of these is essentially a 'spike', the galaxies being located practically at the cluster centres. On the contrary, only $\sim 56 \%$ of the radio-quiet cDs are located within this radius and thus, their intrinsic distributions are likely to be considerably wider.

Could the difference in the location of radio-loud and radio-quiet cDs be a selection effect? Two possible instrumental effects that can cause a loss in detection sensitivity away from cluster centres are, the primary beam attenuation of individual telescopes and, the effect of bandwidth smearing. Most of the $20 \mathrm{~cm}$ maps have a detection threshold for unresolved sources of $\approx 1 \mathrm{mJy}$ at the cluster centres, which increases to $\approx 2 \mathrm{mJy}$ about 15 arcmin away from phase centres. For a mean redshift $\langle z\rangle \approx 0.15$, the Abell radius $R_{A}$ is also $\approx 15$ arcmin. Beam attenuation is therefore unlikely to have a significant effect as all of the radio-loud cDs are detected above the flux density of $2 \mathrm{mJy}$ and the optical positions of all the cDs are within 5 arcmin from centres. Similarly, the effect of bandwidth smearing on radio detection within 5 arcmin from centres is quite small (about $10 \%$ loss in peak sensitivity with total flux density remaining unchanged) and is unlikely to have caused any systematic selection. Thus, the evidence for radio-emitting cDs to be more centrally located in clusters is very unlikely to be a selection effect.

We believe that the more central location of radio-loud $\mathrm{cD}$ galaxies is possibly related to the dynamical evolution of the clusters. In section 6 we have presented evidence for a strong correlation between radio emission from cDs and cooling-flows in their vicinity. All such cooling-flow clusters are known to have centrally peaked and spherically symmetric X-ray brightness distributions (Jones \& Forman 1984). The distribution of galaxies in such clusters also shows a similar trend (Bahcall 1977; Oemler 1974) with higher than average velocity dispersions (Jones \& Forman 1984). This suggests that the cooling-flow clusters are relatively more dynamically evolved with deeper central gravitational potential wells where $\mathrm{a} \mathrm{cD}$ is almost always present. However, not all clusters could have relaxed during the Hubble time in a dynamical model where clusters are products of gravitational growth of small initial density perturbations. In the case of clusters of dimension $R_{G} \approx 1 \mathrm{Mpc}\left(R_{G}\right.$, the gravitational radius), and of mass $\approx 10^{14} M_{\odot}$, the collapse time $t$ coll $\approx 1.5 \times 19^{9} \mathrm{yr}$, whereas for a configuration with ${ }_{A} \approx 10 \mathrm{Mpc}$ and the same mass, the collapse time is $\approx 4.7 \times 10^{10} \mathrm{yr}$ (Sarazin 1988). This means that many of the clusters must be collapsing and merging at the present epoch. McGlynn \& Fabian (1984) point out that during the merger of dense subclusters with each other, any cooling-flow if operating within subsclusters would be completely disrupted. The radio emission from a cD galaxy in the process of evolution in a merging subcluster should then be quenched. This mechanism provides a natural explanation to the phenomenon of radio emission from $\mathrm{cD}$ galaxy being influenced by its location in the cluster.

In order to further check the validity of our hypothesis of radio emission from dominant galaxies in clusters being influenced by the dynamical state of the cluster, 
we have studied the optical morphology of the radio-loud and radio-quiet clusters. The morphological classes of the clusters used by us are obtained from the compilations of Struble \& Rood (1991) who classified the Abell clusters into a 'double-tuning-fork' sequence (the Rood-Sastry or RS sequence) which runs from less evolved/irregular clusters (RS classes, I, F) to intermediate stage (RS classes L, C) and finally to more-evolved/relaxed clusters (RS classes $\mathrm{B}, \mathrm{cD}$ ). In Table 4 we present the data on the fraction of the radio-detected clusters from our sample in each of RS classes. Clearly, this fraction of radio detection is correlated with cluster morphology with $55 \%, 33 \%, 25 \%$ and $0 \%$ being detected among the classes $\mathrm{cD}, \mathrm{B}, \mathrm{L}+\mathrm{C}$ and $\mathrm{F}+\mathrm{I}$ respectively. It should be emphasized here that our cluster sample is selected only on the basis of optical brightness contrast of the brightest cluster member relative to less brighter members (BM types I, I:; Bautz \& Morgan 1970) and thus should be more or less unbiased by the morphological state of the cluster. Despite this, the evidence favours an increasing probability of radio emission from the dominant members of more evolved clusters. This gives us confidence in concluding that, we are observing a real effect of cluster dynamical evolution on radio emission probability, and not an artefact of observational selection effects.

The presently popular cold dark matter theory requires that the clusters evolve towards dynamical relaxation by merging of pre-existing subcluster units. Computer simulations on a large scale appear to reproduce these structures (eg. Evrard 1990; Roettiger et al. 1993). However, the observational evidence for merging subclusters is controversial in optical studies (Geller 1990) but is quite strong in X-ray observations (eg. Jones \& Forman 1984). A recent high sensitivity X-ray study of the Coma cluster with the ROSAT satellite in fact provides evidence for ongoing mergers of large subunits containing cD-like galaxies in their centres (White et al. 1993). It is also of

Table 4. Effect of cluster morphology on radio detection probability.

\begin{tabular}{|c|c|c|c|c|}
\hline \multicolumn{2}{|c|}{$\begin{array}{l}\text { Revised } \\
\text { Rood-Sastry } \\
\text { morphological } \\
\text { class }\end{array}$} & Abell number & \multirow[t]{2}{*}{$\begin{array}{l}\text { Number } \\
\text { surveyed }\end{array}$} & \multirow{2}{*}{$\begin{array}{c}\begin{array}{c}\text { Percentage } \\
\text { radio } \\
\text { detection }\end{array} \\
55 \%\end{array}$} \\
\hline & (cD) & $\begin{array}{l}77,136,146,192,261, \\
360,510,690,733,734, \\
1068,1413,1597,1602, \\
1738,2381,2397,2416, \\
2420,2533\end{array}$ & & \\
\hline Binary & (B) & $\begin{array}{l}22,42,122,214,394 \\
882,1277,1391,1576 \\
2456,2521,2579\end{array}$ & 12 & $33 \%$ \\
\hline $\begin{array}{l}\text { Line } \\
\text { and } \\
\text { Core }\end{array}$ & $\begin{array}{l}\text { (L) } \\
\text { (C) }\end{array}$ & $994,1027,1839,2025$ & 04 & $25 \%$ \\
\hline $\begin{array}{l}\text { Flat } \\
\text { and } \\
\text { Irregula }\end{array}$ & $\begin{array}{l}\text { (F) } \\
\text { (I) }\end{array}$ & $\begin{array}{l}1508,2364,2575,2577, \\
2667\end{array}$ & 05 & $0 \%$ \\
\hline
\end{tabular}


interest to note that many of the best cluster candidates showing observational evidence for recent mergers (eg. A399-A401, A2634, A 2256 and Coma), show an absence of cooling flows (Edge et al. 1992) with weak or no detectable radio emission from their central galaxies (excluding WATs; see section 6). Thus, the available observations of nearby clusters support our hypothesis. Futher confirmation would be to show that the radioloud cDs are preferentially located at the focii of the well developed cooling flow systems and have small relative velocities with respect to the intracluster medium. The existence of subclusters in these clusters should also be rarer compared to radio-quiet ones. An observation of the dynamical states of the radio-loud and radio-quiet clusters (preferentially with an X-ray telescope) and a measurement of the shape and depth of their gravitational potential wells could thus provide a definite answer.

\section{Spectral indices of $\mathrm{cD}$ radio galaxies}

Out of the 41 clusters for which we measured the optical position of the $\mathrm{cD}$ galaxies, only 29 clusters have the radio data at both $20 \mathrm{~cm}$ and $90 \mathrm{~cm}$ wavelength. In these clusters, we have a sample of 14 radio sources either with definite spectral indices (10), or with upper limits (4). A plot of the distribution of $\alpha_{0.3}^{1.4}$ for the 14 sources is shown in Fig 5. Using only the actual detections the median value of $\alpha_{0.3}^{1.4}$ is derived to be $\left(\alpha_{0.3}^{1.4}\right)_{\mathrm{med}}=0.70 \pm 0.13$ and the mean spectral index for this distribution is $\left(\alpha_{0.3}^{1.4}\right)_{\text {mean }}=0.66$. However, assuming the upper limits to represent the actual values, the median spectral index is derived to be $\left(\alpha_{0.3}^{1.4}\right)_{\text {med }}=0.83 \pm 0.13$. These fairly low values of the median spectral indices are somewhat surprising as it is generally believed that there is a significant population of very steep spectrum radio sources (with $\left.\alpha_{0.3}^{1.4}>1.0\right)$ in rich clusters of galaxies. In fact, our estimated median value of $\approx 0.75$

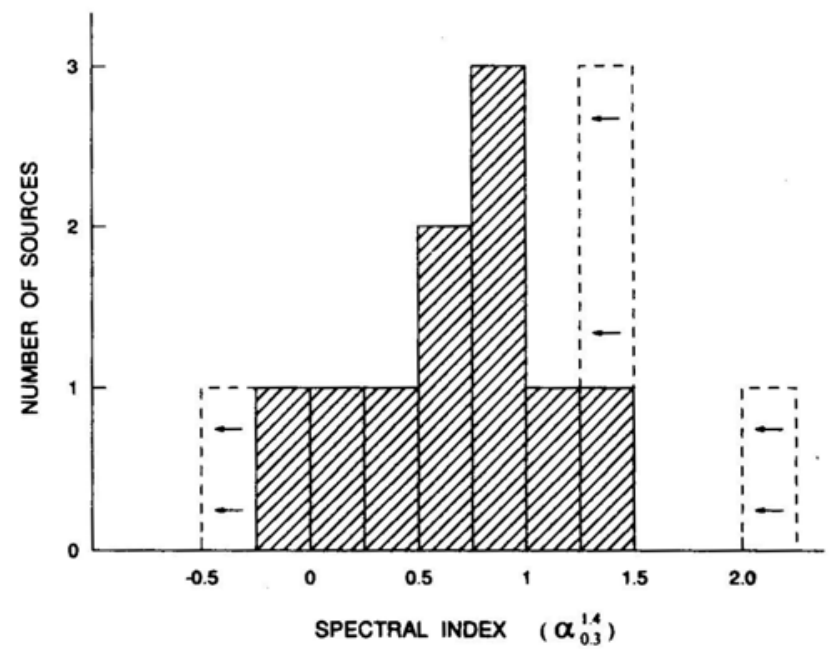

Figure 5. Histogram of the spectral index distribution for $\mathrm{cD}$ galaxies between the frequencies of $1.4 \mathrm{GHz}$ and $330 \mathrm{MHz}$. The dotted sections represent upper limits to the spectral indices. 
is similar to the median spectral index of canonical radio galaxies of similar radio uminosities but not necessarily in rich clusters of galaxies (Laing \& Peacock 1980). An early study by McHardy (1979) reported that the mean radio spectral index of cD galaxies in BM type I clusters was $1.13 \pm 0.24$ (between $1400 \mathrm{MHz}$ and $408 \mathrm{MHz}$ ). This spectrum was found by associating Abell clusters with radio sources selected from 4C survey and subsequently reobserving them at a higher resolution using Cambridge 1-Mile telescope (McHardy 1979). Although both the results, ours as well as McHardy's, are based on a rather small number of objects, the difference needs to be explained as spectral index is an important tool for understanding of the evolution of radio sources. Here we compare the two samples considering certain well known effects which might be responsible for the difference in spectral index distribution in the two samples.

- Spectral index vs selection frequency: McHardy's (1979) sample was selected from a radio survey made at $v=178 \mathrm{MHz}$ which is much lower than the selection frequency of $1.4 \mathrm{GHz}$ in our survey. It is well known that the median spectral index moves towards steeper values as one goes to lower selection frequencies.

- Luminosity vs spectral index correlation: The limiting flux density of $2 \mathrm{Jy}$ for. the $4 \mathrm{C}$ survey implies that from clusters with $\mathrm{z} \approx 0.1$, no radio source with $178 \mathrm{MHz}$ radio luminosity $<8.6 \times 10^{25} \mathrm{WHz}^{-1}$ (or $1.4 \mathrm{GHz}$ luminosity $<1.8 \times 10^{25} \mathrm{WHz}^{-1}$ for $\alpha=0.75$ ) can be detected. The RLF of $\mathrm{cD}$ galaxies detected by us, on the other hand extends down to $\approx 10^{22} \mathrm{WHz}^{-1}$ and we find that only $\sim 5 \%$ of the cDs emit a radio luminosity $>10^{25} \mathrm{WHz}^{-1}$. The cluster sources of McHardy thus are of much higher luminosities (by a factor of $\gtrsim 100$ ) than the typical sources associated with $\mathrm{cDs}$ in our survey. A positive correlation between radio luminosity and the spectral index is well known for radio sources in 3CR catalogue (Laing \& Peacock 1980). This correlation could also be partly responsible for the steeper spectra found by McHardy.

In view of the small number of sources in both the samples, it is not clear if the entire discrepancy in the spectral index distributions can be explained by the above two effects. It is also possible that the occurrence of very steep spectrum sources in BM I clusters is confined only to the high end of the luminosity distribution. The presence of some extremely steep spectrum radio sources $(\alpha \gtrsim 1)$ in clusters of galaxies is however well established (Baldwin \& Scott 1973; Slingo 1974 a, b). Generally, these objects are of two types. The 'relic' radio plasmons of diffuse and extended morphology located near cluster centres but rarely coincident with any cluster member galaxy (eg. Slee \& Reynolds 1984; Joshi et al 1986), and the so called head-tail radio galaxies (eg. O'Dea \& Owen 1986). The underlying cause of the steep spectral indices for both the classes in the same, viz. 'ageing' of relativistic electrons due to radiation losses from a volume that no longer contains an active source of fresh particles: The value of $\left(\alpha_{0.3}^{1.4}\right)_{\text {med }}=0.7$ found by us for $\mathrm{cD}$ galaxies on the other hand implies that this spectral ageing is not visible due to injection of fresh particles from the nuclear active regions into emitting volumes. We now apply the Kardashev (1962) theory in order to better understand the spectral evolution of such sources in the cluster medium.

The radio spectrum of a source evolves in two stages: during the first stage a radio plasmon receives a continuous supply of relativistic particles and expands till it comes to a pressure balance with the external medium. At this phase if $\alpha_{0}$ be the spectral index of the radio spectrum at the time of injection, a 'break' in the spectrum, with index $\alpha_{1}=\alpha_{0}+0.5$ beyond the break frequency develops. In the second stage if the supply of fresh particles ceases due to any reason, a second spectral break with 
spectrum further steepening to index $\alpha_{2}=\left(\frac{4}{3}\right) \alpha_{1}+1$ beyond this break frequency develops. If we identify the average initial spectrum at the time of injection with $\alpha_{0}=0.7$, then $\alpha_{1}=1.2$ and $\alpha_{2}=2.6$. Two of the cDs are detected with $\alpha_{0.3}^{1.4}=1$ to 1.5 and none with $\alpha_{0.3}^{1.4}>1.5$. Thus it can be safely concluded that the second spectral break is not yet visible in any of the sources and only a few may contain the first break. The location of the break in the spectrum is a diagnostic for the elapsed time since the two stages mentioned above. After a time $t_{1}$ the first break would be found at a frequency $v_{1}$. given by the formula (Kardashev 1962, but modified to include inverse Compton losses)

$$
v_{1}(\mathrm{~Hz})=\left(\frac{5.44 \times 10^{9} \times B(\mathrm{Gauss}) \times t_{1}^{-2}(y r)}{\left[B^{2}(\mathrm{Gauss})+B_{\mathrm{MBR}}^{2}(\mathrm{Gauss})\right]^{2}}\right),
$$

where $B$ is the internal magnetic field and $B_{\mathrm{MBR}}=2.57 \times 10^{-6} \times(1+z)^{2}$ Gauss is the effective magnetic field due to microwave background radiation field at the redshift $z$. We do not have the value for $B$ and hence use the commonly found equipartition value of $\sim 10^{-6}$ Gauss. Using $B_{\mathrm{MBR}}=3.4 \times 10^{-6}$ Gauss corresponding to average $\langle z\rangle=0.15$ for our sample and assuming the break frequency to lie above $v_{1}=1400$ $\mathrm{MHz}$ in the majority of cases, an upper limit for the probable spectral age, $t_{1}<1.6$ $\times 10^{8} \mathrm{yr}$, is then obtained. As majority of radio loud cDs are most probably located at. the centres of dense intracluster media of cooling-flow systems (section 6 above), it is possible that the radio emitting volumes do not expand to large sizes and consequently the internal magnetic fields $B<10^{-6}$ Gauss. We can estimate the more probable value of the internal magnetic field if we assume pressure equilibrium with the external medium and the minimum energy condition. Under these assumptions,

$$
B=\left[\frac{72 \cdot \pi}{13} \cdot P_{g a s}\right]^{1 / 2}
$$

If we use the average value for the gas pressure $\left\langle P_{\text {gas }}\right\rangle=4 \times 10^{-11} \mathrm{dyn} \mathrm{cm}^{-2}$ obtained from the sample of clusters with cooling flows studied in section 6 , we then obtain $B=2.6 \times 10^{-5}$ Gauss and an even shorter spectral age of $t_{1}<1.5 \times 10^{7} \mathrm{yr}$. Higher resolution radio observations of the detected sources to actually detect active cores and X-ray data on the state of intracluster media is required to confirm these numbers.

In conclusion, our observations for the spectral indices of these $\mathrm{cD}$ galaxies indicate them to be relatively 'young' in terms of their spectral ages. The evidence favours an ongoing activity in the central regions of many of them and if located at the centres of dense cluster media of cooling flow systems, the spectral ages come out to be $<2 \times 10^{7} \mathrm{yr}$ for the majority of them.

\section{Summary and conclusions}

We have presented the results of a relatively deep radio survey at $20 \mathrm{~cm}$ and $90 \mathrm{~cm} \lambda$ of a Statistical sample of distant Abell clusters $(0.1 \lesssim z \lesssim 0.3)$ containing dominant $\mathrm{cD}$ galaxies. The important results and conclusions from this study can be summarized as follows: 
- The radio luminosity function for the $\mathrm{cD}$ galaxies appears to agree quantitatively with the RLF of brightest giant elliptical galaxies (located in less rich galactic environments) for $1.4 \mathrm{GHz}$ radio luminosity $\leqslant 10^{24.5} \mathrm{WHz}^{-1}$. At higher luminosities the $\mathrm{cD}$ galaxies appear to be more likely to be radio sources. This behaviour of the RLF can be understood in terms of the correlation between the radio and optical luminosities of the giant elliptical systems, and suggests a unified mechanism for the triggering of the nuclear activity in radio galaxies in diverse environments.

- We do not detect a significantly large population of very steep spectrum radio sources with $\alpha>1.2$ (between $1.4 \mathrm{GHz}$ and $0.3 \mathrm{GHz}$ ) to be associated with the cD galaxies. The median spectral index is found to be only 0.70 , similar to that of typical radio galaxies not necessarily in rich clusters. The evidence favours ongoing activity in the central regions of many of the $\mathrm{cD}$ galaxies, and if they are located at the centres of the dense cluster media of cooling-flow systems, the spectral ages are likely to be $<2 \times 10^{7}$ y for the majority of them.

- An examination of the existing radio and X-ray data on the nearby $(0.03<z<0.15)$ Abell clusters with $\mathrm{cD}$ galaxies has revealed a significant inverse correlation between the radio luminosity of the $\mathrm{cD}$ galaxy and the cooling time of the hot intra-cluster medium in its vicinity. As many as $70 \%$ of the radio emitting $\mathrm{cD}$ galaxies (with $1.4 \mathrm{GHz}$ radio luminosity $\gtrsim 10^{22.7} \mathrm{WHz}^{-1}$ ) are found to be located at the focii of cooling flows and this suggests the presence of cooling flows in many of the distant Abell clusters containing radio-loud $\mathrm{cD}$ galaxies in our sample. In order to better understand the observed effect we have considered the physical models of confinement of the radio plasmas and the fuelling of the central energy sources by means of accretion. It is concluded that though confinement is necessary, it may not be a sufficient factor. The evidence currently available supports the idea that nuclear activity is triggered by the accretion of the cooler gas by the massive galaxies forming at the centres of cooling flows in dynamically evolving clusters of galaxies.

- We have found evidence for the probability of radio emission from cDs to be related to their location within the cluster. The radio emitting $\mathrm{cDs}$ are located close to the centres of their host clusters, as against the radio-quiet ones, which are less centrally concentrated. That this could be related to the dynamical evolution of clusters is supported also by our observation that, even though all the clusters selected for our study had a first ranked giant elliptical galaxy, the probability of radio emission from this galaxy was found to be correlated with the evolutionary morphological class (the Rood-Sastry class) of the parent cluster. Dynamically more evolved classes of clusters $(\mathrm{cD}, \mathrm{B})$ are more likely to have radio sources associated with the brightest clusters members as against less evolved, irregular morphological classes $(\mathrm{L}, \mathrm{C}, \mathrm{F}, \mathrm{I})$. We have argued that this effect together with the correlation between the cooling time and the radio luminosity mentioned above indicates that the phenomenon of radio emission from the central galaxies in rich Abell clusters is mainly governed by the effects of the dynamical evolution of the clusters.

\section{Acknowledgement}

We gratefully acknowledge the support, help and advice from the late Prof. M. N. Joshi. We thank Dr. J. P. Huchra for providing us spectroscopic redshifts for many of the clusters in our sample. We have made extensive use of the computational and 
other facilities at the Radio Astronomy Centre, Ootacamund and at TIFR Bombay. The Very Large Array is operated by Associated Universities Inc., under contract with the National Science Foundation, USA.

\section{References}

Abell, G. O. 1958, Astrophys. J. Suppl. 3, 211.

Abell, G. O., Corwin, H. G., Olowin/R. P. 1989, Astrophys. J. Suppl. 70, 1.

Albert, C. E., White, R. A., Morgan, W. W. 1977, Astrophys. J. 211, 309.

Auriemma, C, Perola, G. C, Ekers, R., Fanti, R., Lari, C, Jaffe, W. J., Ulrich, M. H. 1977, Astr. Astrophys. 57,41.

Baars, J. W. M., Genzel, R., Pauliny-Toth, I.I. K., Witzel, A. 1977, Astr. Astrophys. 61, 99.

Bahcall, N. A. 1977, A. Rev. Astr. Astrophys., 15, 505.

Baldwin, J. E., Scott, P. F. 1973, Mon. Not. R. astr. Soc. 165, 259.

Ball, R., Bums, J. O., Loken, C. 1993, Astr. J., 105, 53.

Bautz, L. P., Morgan, W. W. 1970, Astrophys. J. Lett., 162, L149.

Begelman, M. C, Blandford, R. D., Rees, M. J. 1984, Rev. Mod. Phys., 56, 255.

Burns, J. O. 1990, Astr. J., 99, 14.

Colla, G., Fanti, C, Gioia, I, Lari, C. 1975, Astr. Astrophys., 38, 209.

Crawford, C. S., Fabian, A. C, Johnstone, R. M. 1988, Mon. Not. R. astr. Soc, 235, 183.

Crawford, C. S., Fabian, A. C. 1989, Mon. Not. R. astr. Soc, 239, 219.

Donahue, M., Stocke, J. J., Gioia, I. M. 1992, Astrophys. J. 385, 49.

Dressier, A. 1980, Astrophys. J. Suppl., 42, 565.

Edge, A. C, Stewart, G. C, Fabian, A. C. 1992, Mon. Not. R. astr. Soc, 258, 177.

Evrard, A. E. 1990, Astrophys. J., 363, 349.

Fabian, A. C, Nulsen, P. E. J. Canizares, C. R. 1984, Nature, 310, 733.

Fabian, A. C. (Ed) 1987, Cooling Flows in Clusters and Galaxies (Dordrecht: Kluwer Acad. Pub.).

Fabian, A. C, Crawford, C. S. 1990, Preprint.

Fabian, A. C, Nulsen, P. E. J., Canizares, C. R. 1991, Astr. Astrophys. Rev., 2, 191.

Geller, M. 1990, in Proc. of Clusters of Galaxies Meeting (Cambridge Univ. Press), pp. 25.

Gopal-Krishna, Wiita, P. J. 1991, Astrophys. J., 373, 325.

Gunn, J. E., Gott, J. R. 1972, Astrophys. J., 176, 1.

Gunn, J. E., Hoessel, J. G., Oke, J. B. 1986, Astrophys. J., 306, 30.

Harris, D. E., Lari, C, Valee, J. P., Wilson, A. S. 1980, Astr. Astrophys. Suppl, 42, 319.

Hubble, E. P. 1936, in The Realm of the Nebulae (New Haven: Yale Univ. Press), pp. 210.

Huchra, J. P., Henry, J. P., Postman, M., Geller, M. J. 1990, Astrophys. J., 365, 66.

Hutchings, J. B., Crampton, D., Campbell, B. 1981, Astrophys. J., 247, 743.

Jones, C, Forman, W. 1984, in Clusters and Groups of Galaxies (Dordrecht: D. Reidel Pub Comp.), pp. 319.

Joshi, M. N., Kapahi, V. K., Bagchi, J. 1986, in Proc. of the NRAO Workshop on 'Radio Continuum Processes in Clusters of Galaxies, (Eds.) C. P. O, Dea \& J. M. Uson, (NRAO) pp. 73.

Kardashev, N. S. 1962, Astr. Zh, 39, 393.

Kapahi, V. K. 1989, Astr. J., 97, 1.

Kriss.G. A, Cioffi, D. F., Canizares, C. R. 1983, Astrophys. J., 272, 429.

Laing, R. A., Peacock, J. A. 1980, Mon. Not. R. astr. Soc, 190, 903.

Leir, A. A., van Den Bergh, S. 1977. Astrophys. J. Suppl, 34, 381.

Mathew, T. A., Morgan, W. W., Schmidt, M. 1964, Astrophys. J., 140, 35.

McGlynn, T. A., Fabian, A. C. 1984, Mon. Not. R. astr. Soc, 208, 709.

McHardy, I. M. 1979, Mon. Not. R. astr. Soc, 188, 495.

Melnick, J, Sargent, W. L. W. 1977, Astrophys. J., 215, 401.

Morgan, W. W., Lesh, J. 1965, Astrophys. J., 142, 1364.

Morgan, W. W., Kayser, S., While, R. A. 1975, Astrophys. J., 199, 545.

Napier, P. J., Thompson, A. R., Ekers, R. D. 1983, Proc IEEE, 71,1295.

O' Dea, C. P., Owen, F. N. 1986, Astrophys. J., 301, 841. 
Oemler, A. Jr. 1974, Astrophys. J., 194, 1.

Owen, F. N., Burns, J. O., White, R. A. 1984, in Clusters and Groups of Galaxies (Dordrecht: D. Reidel Pub. Comp.), pp. 295.

Roettiger, K., Burns, J., Loken, C. 1993, Astrophys. J. Lett., 407, L53.

Ryle, M. 1968, A. Rev. Astr. Astrophys., 6, 249.

Sandage, A. 1976, Astrophys. J., 205, 6.

Sarazin, C. L. 1988, in X-ray Emissions from Clusters of Galaxies (Cambridge Univ. Press) p. 33.

Schmidt, M. 1968, Astrophys. J., 151, 393.

Schwartz. D. A., Bradt, H. V., Remillard, R. A. 1991, Astrophys. J., 376, 424.

Slee, O. B., Reynolds, J. E. 1984, Proc. astr. Soc. Australia, 5, 516.

Slingo, A. 1974(a), Mon. Not. R. astr. Soc, 166, 101.

Slingo, A. 1974(b), Mon. Not. R. astr. Soc, 168, 307.

Struble, M. F., Rood, H. J. 1991, Astrophys. J. SuppL, 77, 363.

Valentijn, E. A., Bijleveld, W. 1983, Astr. Astrophys., 125, 223.

Yee, H. K. C, Green, R. F. 1987, Astrophys. J., 319, 28.

White, S. D. M, Briel, U. G., Henry, J. P. 1993, Mon. Not. R. astr. Soc, 261, L8.

Zhao, J. H., Burns, J. O., Owen, F. N. 1989, Astr. J., 98, 64.

Zwicky, F. 1938, Publ. astr. Soc. Pacific, 50, 218. 